\title{
Research Article \\ Some Sequential Boundary Crossing Results for Geometric Brownian Motion and Their Applications in Financial Engineering
}

\author{
Tristan Guillaume \\ Laboratoire Thema, Université de Cergy-Pontoise, 33 boulevard du port, \\ 95011 Cergy-Pontoise Cedex, France \\ Correspondence should be addressed to Tristan Guillaume, tristan.guillaume@u-cergy.fr
}

Received 21 March 2011; Accepted 12 April 2011

Academic Editors: L. Ju and M. Skliar

Copyright (C) 2011 Tristan Guillaume. This is an open access article distributed under the Creative Commons Attribution License, which permits unrestricted use, distribution, and reproduction in any medium, provided the original work is properly cited.

This paper provides new explicit results for some boundary crossing distributions in a multidimensional geometric Brownian motion framework when the boundary is a piecewise constant function of time. Among their various possible applications, they enable accurate and efficient analytical valuation of a large number of option contracts traded in the financial markets belonging to the classes of barrier and look-back options.

\section{Introduction}

The joint law of the maximum (or the minimum) of a real-valued Brownian motion and its endpoint over a finite time interval is a central result in the study of Brownian motion, particularly with regard to the many applications of the theory in finance, medical imaging, robotics, and biology. It can be obtained as a consequence of the "reflection principle," which derives from the strong Markov property of Brownian motion (Freedman [1]). Application of Girsanov's theorem easily generalizes this seminal result to the case of a geometric Brownian motion (GBM), a frequently encountered diffusion process that is the building block of financial engineering. Alternatively, the law can be derived by a partial differential equation approach, using Kolmogorov's equation for the transition density function of a diffusion process. The distribution of the first passage time by a one-dimensional GBM to a onesided or a two-sided straight boundary then follows. A few cases where the boundary is curved have been handled (Barba Escribá [2]; Salminen [3]; Kunitomo and Ikeda [4]). For a comprehensive source of formulae, one may refer to Borodin and Salminen [5].

Various extensions to these results are often needed to solve practical engineering problems. In particular, one may look for joint distributions of the highest or lowest points 
hit over several time intervals, and one may deal with a multidimensional GBM. There are few known explicit results in these more general settings, partly because of the laborious analytical calculations involved, but also due to numerical obstacles: the rapidly increasing dimension of the boundary crossing problem leads to analytical solutions that are expressed in terms of functions that are hard to compute with accuracy. As far as one-sided boundaries are concerned, formulae have been published for the joint law of a sequence of maxima or minima of a one-dimensional GBM over several time intervals (Guillaume [6]), as well as for the joint law of the maxima or minima of a two-dimensional GBM over one time interval (Iyengar [7]; He et al. [8]). A formula for the joint law of the exit times of a one-dimensional GBM from two successive two-sided boundaries is also known (Guillaume [9]).

This paper focuses on a sequence of two one-sided straight boundaries conditional on two correlated GBMs, while the value of a third correlated GBM is taken into account at the endpoint of the time interval. The state space is thus three-dimensional. The choice of this particular distribution is motivated both by its usefulness in financial engineering applications and by the fact that it leads to tractable analytical solutions that can be computed with great accuracy and efficiency.

Section 2 presents the two main formulae of this paper. Section 3 deals with applications of these formulae and discusses their numerical implementation.

\section{Main Formulae}

This section contains the two main formulae of the paper. Let $\left\{S_{1}(t), t \geq 0\right\}$ and $\left\{S_{2}(t), t \geq 0\right\}$ be two geometric Brownian motions with constant drift coefficients, $\alpha_{1}$ and $\alpha_{2}$, respectively, under a probability measure $\mathbb{P}$, constant positive diffusion coefficients $\sigma_{1}$ and $\sigma_{2}$, respectively, and constant correlation coefficient $\rho_{1 \cdot 2}$. In other words, the processes $S_{1}(t)$ and $S_{2}(t)$ evolve in time according to the following dynamics:

$$
\begin{aligned}
& d S_{1}(t)=\alpha_{1} S_{1}(t) d t+\sigma_{1} S_{1}(t) d B_{1}(t), \\
& d S_{2}(t)=\alpha_{2} S_{2}(t) d t+\sigma_{2} S_{2}(t) d B_{2}(t),
\end{aligned}
$$

where $B_{1}(t)$ and $B_{2}(t)$ are standard real-valued Brownian motions and $d\left[B_{1}, B_{2}\right](t)=\rho_{1 \cdot 2} d t$. Let $H_{1}, H_{2}, K_{1}, K_{2}, K_{3}$ be positive real numbers and $P_{[\cdot]}\left(H_{1}, H_{2}, K_{1}, K_{2}, K_{3}, t_{1}, t_{2}\right)$ defined as one of the four following cumulative distribution functions, where $t_{2} \geq t_{1} \geq t_{0}=0$ :

$$
\begin{aligned}
& P_{\text {[up-and-up] }}\left(H_{1}, H_{2}, K_{1}, K_{2}, K_{3}, t_{1}, t_{2}\right) \\
& \quad \triangleq \mathbb{P}\left(\sup _{0 \leq t \leq t_{1}} S_{1}(t) \leq H_{1}, S_{1}\left(t_{1}\right) \leq K_{1}, S_{2}\left(t_{1}\right) \leq K_{2}, \sup _{t_{1} \leq t \leq t_{2}} S_{2}(t) \leq H_{2}, S_{2}\left(t_{2}\right) \leq K_{3}\right),
\end{aligned}
$$

(2)

$$
\begin{aligned}
& P_{\text {[down-and-down] }}\left(H_{1}, H_{2}, K_{1}, K_{2}, K_{3}, t_{1}, t_{2}\right) \\
& \quad \triangleq \mathbb{P}\left(\inf _{0 \leq t \leq t_{1}} S_{1}(t) \geq H_{1}, S_{1}\left(t_{1}\right) \geq K_{1}, S_{2}\left(t_{1}\right) \geq K_{2}, \inf _{t_{1} \leq t \leq t_{2}} S_{2}(t) \geq H_{2}, S_{2}\left(t_{2}\right) \geq K_{3}\right),
\end{aligned}
$$


(3)

$$
\begin{aligned}
& P_{\text {[down-and-up] }}\left(H_{1}, H_{2}, K_{1}, K_{2}, K_{3}, t_{1}, t_{2}\right) \\
& \quad \triangleq \mathbb{P}\left(\inf _{0 \leq t \leq t_{1}} S_{1}(t) \geq H_{1}, S_{1}\left(t_{1}\right) \geq K_{1}, S_{2}\left(t_{1}\right) \leq K_{2}, \sup _{t_{1} \leq t \leq t_{2}} S_{2}(t) \leq H_{2}, S_{2}\left(t_{2}\right) \leq K_{3}\right),
\end{aligned}
$$

(4)

$$
\begin{aligned}
& P_{\text {[up-and-down] }}\left(H_{1}, H_{2}, K_{1}, K_{2}, K_{3}, t_{1}, t_{2}\right) \\
& \quad \triangleq \mathbb{P}\left(\sup _{0 \leq t \leq t_{1}} S_{1}(t) \leq H_{1}, S_{1}\left(t_{1}\right) \leq K_{1}, S_{2}\left(t_{1}\right) \geq K_{2}, \inf _{t_{1} \leq t \leq t_{2}} S_{2}(t) \geq H_{2}, S_{2}\left(t_{2}\right) \geq K_{3}\right) .
\end{aligned}
$$

Let us introduce the following notations:

$$
\begin{gathered}
k_{1}=\ln \left(\frac{K_{1}}{S_{1}\left(t_{0}\right)}\right), \quad k_{2}=\ln \left(\frac{K_{2}}{S_{2}\left(t_{0}\right)}\right), \quad k_{3}=\ln \left(\frac{K_{3}}{S_{2}\left(t_{0}\right)}\right), \\
h_{1}=\ln \left(\frac{H_{1}}{S_{1}\left(t_{0}\right)}\right), \quad h_{2}=\ln \left(\frac{H_{2}}{S_{2}\left(t_{0}\right)}\right), \\
\mu_{1}=\alpha_{1}-\frac{\sigma_{1}^{2}}{2}, \quad \mu_{2}=\alpha_{2}-\frac{\sigma_{2}^{2}}{2} .
\end{gathered}
$$

The next proposition provides an exact formula for the four above-mentioned cumulative distribution functions.

Proposition 2.1. Let $N_{3}\left[\cdot, \cdot, \cdot ; \theta_{1 \cdot 2}, \theta_{1 \cdot 3}, \theta_{2 \cdot 3}\right]$ denote the joint trivariate cumulative distribution function of three standard normal random variables $X_{1}, X_{2}, X_{3}$, where $\theta_{a \cdot b}$ is the correlation coefficient between $X_{a}$ and $X_{b},(a, b) \in\{1,2,3\}$.

Then, for the up-and-up and the down-and-down distributions, one has

$$
\begin{aligned}
& P_{[\cdot]}\left(H_{1}, H_{2}, K_{1}, K_{2}, K_{3}, t_{1}, t_{2}\right) \\
&=N_{3}\left[\lambda\left(\frac{k_{1}-\mu_{1} t_{1}}{\sigma_{1} \sqrt{t_{1}}}\right), \lambda\left(\frac{k_{2}-\mu_{2} t_{1}}{\sigma_{2} \sqrt{t_{1}}}\right), \lambda\left(\frac{k_{3}-\mu_{2} t_{2}}{\sigma_{2} \sqrt{t_{2}}}\right) ; \rho_{1 \cdot 2}, \sqrt{\frac{t_{1}}{t_{2}}} \rho_{1 \cdot 2}, \sqrt{\frac{t_{1}}{t_{2}}}\right] \\
&-\exp \left(\frac{2 \mu_{2} h_{2}}{\sigma_{2}^{2}}\right) N_{3}\left[\begin{array}{l}
\lambda\left(\frac{k_{1}-\mu_{1} t_{1}}{\sigma_{1} \sqrt{t_{1}}}\right)+\rho_{1 \cdot 2} \frac{2 \mu_{2} \sqrt{t_{1}}}{\sigma_{2}}, \lambda\left(\frac{k_{2}+\mu_{2} t_{1}}{\sigma_{2} \sqrt{t_{1}}}\right), \lambda\left(\frac{k_{3}-2 h_{2}-\mu_{2} t_{2}}{\sigma_{2} \sqrt{t_{2}}}\right) ; \\
\rho_{1 \cdot 2},-\rho_{1 \cdot 2} \sqrt{\frac{t_{1}}{t_{2}}},-\sqrt{\frac{t_{1}}{t_{2}}}
\end{array}\right] \\
&-\exp \left(\frac{2 \mu_{1} h_{1}}{\sigma_{1}^{2}}\right)
\end{aligned}
$$




$$
\begin{aligned}
& \times N_{3}\left[\begin{array}{l}
\lambda\left(\frac{k_{1}-2 h_{1}-\mu_{1} t_{1}}{\sigma_{1} \sqrt{t_{1}}}\right), \lambda\left(\frac{k_{2}-\mu_{2} t_{1}}{\sigma_{2} \sqrt{t_{1}}}-\rho_{1 \cdot 2} \frac{2 h_{1}}{\sigma_{1} \sqrt{t_{1}}}\right), \lambda\left(\frac{k_{3}-\mu_{2} t_{2}}{\sigma_{2} \sqrt{t_{2}}}-\rho_{1 \cdot 2} \frac{2 h_{1}}{\sigma_{1} \sqrt{t_{2}}}\right) ; \\
\rho_{1 \cdot 2}, \rho_{1 \cdot 2} \sqrt{\frac{t_{1}}{t_{2}}}, \sqrt{\frac{t_{1}}{t_{2}}}
\end{array}\right] \\
& +\exp \left(\left(\frac{2 \mu_{1}}{\sigma_{1}^{2}}-\frac{4 \mu_{2} \rho_{1 \cdot 2}}{\sigma_{1} \sigma_{2}}\right) h_{1}+\frac{2 \mu_{2} h_{2}}{\sigma_{2}^{2}}\right) \\
& \times N_{3}\left[\begin{array}{l}
\lambda\left(\frac{k_{1}-2 h_{1}-\mu_{1} t_{1}}{\sigma_{1} \sqrt{t_{1}}}\right)+\rho_{1 \cdot 2} \frac{2 \mu_{2} \sqrt{t_{1}}}{\sigma_{2}}, \lambda\left(\frac{k_{2}+\mu_{2} t_{1}}{\sigma_{2} \sqrt{t_{1}}}-\rho_{1 \cdot 2} \frac{2 h_{1}}{\sigma_{1} \sqrt{t_{1}}}\right), \\
\lambda\left(\frac{k_{3}-2 h_{2}-\mu_{2} t_{2}}{\sigma_{2} \sqrt{t_{2}}}+\rho_{1 \cdot 2} \frac{2 h_{1}}{\sigma_{1} \sqrt{t_{2}}}\right) ; \rho_{1 \cdot 2},-\rho_{1 \cdot 2} \sqrt{\frac{t_{1}}{t_{2}}},-\sqrt{\frac{t_{1}}{t_{2}}}
\end{array}\right],
\end{aligned}
$$

where

$$
\begin{gathered}
\lambda=1 \quad \text { if } P_{[\cdot]}\left(H_{1}, H_{2}, K_{1}, K_{2}, K_{3}, t_{1}, t_{2}\right)=P_{[\text {[up-and-up }]}\left(H_{1}, H_{2}, K_{1}, K_{2}, K_{3}, t_{1}, t_{2}\right), \\
\lambda=-1 \quad \text { if } P_{[\cdot]}\left(H_{1}, H_{2}, K_{1}, K_{2}, K_{3}, t_{1}, t_{2}\right)=P_{[\text {down-and-down }]}\left(H_{1}, H_{2}, K_{1}, K_{2}, K_{3}, t_{1}, t_{2}\right) .
\end{gathered}
$$

And, for the up-and-down and the down-and-up distributions, one has

$$
\begin{aligned}
& P_{[\cdot]}\left(H_{1}, H_{2}, K_{1}, K_{2}, K_{3}, t_{1}, t_{2}\right) \\
& =N_{3}\left[\lambda\left(\frac{k_{1}-\mu_{1} t_{1}}{\sigma_{1} \sqrt{t_{1}}}\right), \lambda\left(\frac{-k_{2}+\mu_{2} t_{1}}{\sigma_{2} \sqrt{t_{1}}}\right), \lambda\left(\frac{-k_{3}+\mu_{2} t_{2}}{\sigma_{2} \sqrt{t_{2}}}\right) ;-\rho_{1 \cdot 2},-\sqrt{\frac{t_{1}}{t_{2}}} \rho_{1 \cdot 2}, \sqrt{\frac{t_{1}}{t_{2}}}\right] \\
& -\exp \left(\frac{2 \mu_{2} h_{2}}{\sigma_{2}^{2}}\right) \\
& \times N_{3}\left[\begin{array}{l}
\lambda\left(\frac{k_{1}-\mu_{1} t_{1}}{\sigma_{1} \sqrt{t_{1}}}\right)+\rho_{1 \cdot 2} \frac{2 \mu_{2} \sqrt{t_{1}}}{\sigma_{2}}, \lambda\left(\frac{-k_{2}-\mu_{2} t_{1}}{\sigma_{2} \sqrt{t_{1}}}\right), \lambda\left(\frac{-k_{3}+2 h_{2}+\mu_{2} t_{2}}{\sigma_{2} \sqrt{t_{2}}}\right) ; \\
-\rho_{1 \cdot 2}, \sqrt{\frac{t_{1}}{t_{2}}} \rho_{1 \cdot 2},-\sqrt{\frac{t_{1}}{t_{2}}}
\end{array}\right] \\
& -\exp \left(\frac{2 \mu_{1} h_{1}}{\sigma_{1}^{2}}\right) \\
& \times N_{3}\left[\begin{array}{l}
\lambda\left(\frac{k_{1}-2 h_{1}-\mu_{1} t_{1}}{\sigma_{1} \sqrt{t_{1}}}\right), \lambda\left(\frac{-k_{2}+\mu_{2} t_{1}}{\sigma_{2} \sqrt{t_{1}}}+\rho_{1 \cdot 2} \frac{2 h_{1}}{\sigma_{1} \sqrt{t_{1}}}\right), \lambda\left(\frac{-k_{3}+\mu_{2} t_{2}}{\sigma_{2} \sqrt{t_{2}}}+\rho_{1 \cdot 2} \frac{2 h_{1}}{\sigma_{1} \sqrt{t_{2}}}\right) ; \\
-\rho_{1 \cdot 2},-\sqrt{\frac{t_{1}}{t_{2}}} \rho_{1 \cdot 2}, \sqrt{\frac{t_{1}}{t_{2}}}
\end{array}\right]
\end{aligned}
$$




$$
\begin{aligned}
& +\exp \left(\left(\frac{2 \mu_{1}}{\sigma_{1}^{2}}-\frac{4 \mu_{2} \rho_{1 \cdot 2}}{\sigma_{1} \sigma_{2}}\right) h_{1}+\frac{2 \mu_{2} h_{2}}{\sigma_{2}^{2}}\right) \\
& \times N_{3}\left[\begin{array}{l}
\lambda\left(\frac{k_{1}-2 h_{1}-\mu_{1} t_{1}}{\sigma_{1} \sqrt{t_{1}}}\right)+\rho_{1 \cdot 2} \frac{2 \mu_{2} \sqrt{t_{1}}}{\sigma_{2}}, \lambda\left(\frac{-k_{2}-\mu_{2} t_{1}}{\sigma_{2} \sqrt{t_{1}}}+\rho_{1 \cdot 2} \frac{2 h_{1}}{\sigma_{1} \sqrt{t_{1}}}\right), \\
\lambda\left(\frac{-k_{3}+2 h_{2}+\mu_{2} t_{2}}{\sigma_{2} \sqrt{t_{2}}}-\rho_{1 \cdot 2} \frac{2 h_{1}}{\sigma_{1} \sqrt{t_{2}}}\right) ;-\rho_{1 \cdot 2}, \sqrt{\frac{t_{1}}{t_{2}}} \rho_{1 \cdot 2},-\sqrt{\frac{t_{1}}{t_{2}}}
\end{array}\right],
\end{aligned}
$$

where

$$
\begin{aligned}
& \lambda=1 \quad \text { if } P_{[\cdot]}\left(H_{1}, H_{2}, K_{1}, K_{2}, K_{3}, t_{1}, t_{2}\right)=P_{[\text {[up-and-down }]}\left(H_{1}, H_{2}, K_{1}, K_{2}, K_{3}, t_{1}, t_{2}\right), \\
& \lambda=-1 \text { if } P_{[\cdot]}\left(H_{1}, H_{2}, K_{1}, K_{2}, K_{3}, t_{1}, t_{2}\right)=P_{[\text {down-and-up }]}\left(H_{1}, H_{2}, K_{1}, K_{2}, K_{3}, t_{1}, t_{2}\right) .
\end{aligned}
$$

\section{Corollary of Proposition 2.1}

The four following joint cumulative distribution functions, that will be useful in Section 3, are deduced from Proposition 2.1:

(1)

$$
\begin{gathered}
\mathbb{P}\left(\sup _{0 \leq t \leq t_{1}} S_{1}(t) \leq H_{1}, S_{1}\left(t_{1}\right) \leq K_{1}, S_{2}\left(t_{1}\right) \leq K_{2}, \sup _{t_{1} \leq t \leq t_{2}} S_{2}(t) \leq H_{2}, S_{2}\left(t_{2}\right) \geq K_{3}\right) \\
=P_{\text {[up-and-up] }}\left(H_{1}, H_{2}, K_{1}, K_{2}, \infty\right)-P_{\text {[up-and-up] }}\left(H_{1}, H_{2}, K_{1}, K_{2}, K_{3}\right),
\end{gathered}
$$

(2)

$$
\begin{aligned}
& \mathbb{P}\left(\inf _{0 \leq t \leq t_{1}} S_{1}(t) \geq H_{1}, S_{1}\left(t_{1}\right) \geq K_{1}, S_{2}\left(t_{1}\right) \geq K_{2}, \inf _{t_{1} \leq t \leq t_{2}} S_{2}(t) \geq H_{2}, S_{2}\left(t_{2}\right) \leq K_{3}\right) \\
& \quad=P_{\text {[down-and-down] }}\left(H_{1}, H_{2}, K_{1}, K_{2},-\infty\right)-P_{\text {[down-and-down] }}\left(H_{1}, H_{2}, K_{1}, K_{2}, K_{3}\right),
\end{aligned}
$$

(3)

$$
\begin{gathered}
\mathbb{P}\left(\inf _{0 \leq t \leq t_{1}} S_{1}(t) \geq H_{1}, S_{1}\left(t_{1}\right) \geq K_{1}, S_{2}\left(t_{1}\right) \leq K_{2}, \sup _{t_{1} \leq t \leq t_{2}} S_{2}(t) \leq H_{2}, S_{2}\left(t_{2}\right) \geq K_{3}\right) \\
=P_{\text {[down-and-up] }}\left(H_{1}, H_{2}, K_{1}, K_{2}, \infty\right)-P_{\text {[down-and-up] }}\left(H_{1}, H_{2}, K_{1}, K_{2}, K_{3}\right),
\end{gathered}
$$


(4)

$$
\begin{gathered}
\mathbb{P}\left(\sup _{0 \leq t \leq t_{1}} S_{1}(t) \leq H_{1}, S_{1}\left(t_{1}\right) \leq K_{1}, S_{2}\left(t_{1}\right) \geq K_{2} \inf _{t_{1} \leq t \leq t_{2}} S_{2}(t) \geq H_{2}, S_{2}\left(t_{2}\right) \leq K_{3}\right) \\
\quad=P_{\text {[up-and-down] }}\left(H_{1}, H_{2}, K_{1}, K_{2},-\infty\right)-P_{\text {[up-and-down] }}\left(H_{1}, H_{2}, K_{1}, K_{2}, K_{3}\right) .
\end{gathered}
$$

Proof of Proposition 2.1 is provided in the Appendices A, B, and C.

The numerical implementation of Proposition 2.1 and its corollary is easy using Genz's algorithm for the computation of trivariate normal cumulative distribution functions (Genz [10]).

In the next Proposition, we introduce a third correlated geometric Brownian motion that will serve as the endpoint of the joint distribution, and we show that this can still be analytically valued. Let $\left\{S_{3}(t), t \geq 0\right\}$ be a geometric Brownian motion driven by the following dynamics under the initial probability measure $\mathbb{P}$ :

$$
d S_{3}(t)=\alpha_{3} S_{3}(t) d t+\sigma_{3} S_{3}(t) d B_{3}(t)
$$

where $\alpha_{3}$ and $\sigma_{3}$ are real constants $\left(\sigma_{3}>0\right), B_{3}(t)$ is a real-valued standard Brownian motion and correlations are as follows:

$$
d\left[B_{1}, B_{3}\right](t)=\rho_{1 \cdot 3} d t, \quad d\left[B_{2}, B_{3}\right](t)=\rho_{2 \cdot 3} d t
$$

Let $K_{4}$ be a positive real number and $P_{[\cdot]}\left(H_{1}, H_{2}, K_{1}, K_{2}, K_{3}, K_{4}, t_{1}, t_{2}, t_{3}\right)$ defined as one of the four following joint cumulative distribution functions, where $t_{3} \geq t_{2}$ :

(1)

$$
\begin{aligned}
& P_{\text {[up-and-up] }}\left(H_{1}, H_{2}, K_{1}, K_{2}, K_{3}, K_{4}, t_{1}, t_{2}, t_{3}\right) \\
& \quad \triangleq \mathbb{P}\left(\sup _{0 \leq t \leq t_{1}} S_{1}(t) \leq H_{1}, S_{1}\left(t_{1}\right) \leq K_{1}, S_{2}\left(t_{1}\right) \leq K_{2}, \sup _{t_{1} \leq t \leq t_{2}} S_{2}(t) \leq H_{2}, S_{2}\left(t_{2}\right) \leq K_{3}, S_{3}\left(t_{3}\right) \leq K_{4}\right),
\end{aligned}
$$

(2)

$$
\begin{aligned}
& P_{\text {[down-and-down] }}\left(H_{1}, H_{2}, K_{1}, K_{2}, K_{3}, K_{4}, t_{1}, t_{2}, t_{3}\right) \\
& \triangleq \mathbb{P}\left(\inf _{0 \leq t \leq t_{1}} S_{1}(t) \geq H_{1}, S_{1}\left(t_{1}\right) \geq K_{1}, S_{2}\left(t_{1}\right) \geq K_{2}, \inf _{t_{1} \leq t \leq t_{2}} S_{2}(t) \geq H_{2}, S_{2}\left(t_{2}\right) \geq K_{3}, S_{3}\left(t_{3}\right) \geq K_{4}\right),
\end{aligned}
$$


(3)

$$
\begin{aligned}
& P_{\text {[down-and-up] }}\left(H_{1}, H_{2}, K_{1}, K_{2}, K_{3}, K_{4}, t_{1}, t_{2}, t_{3}\right) \\
& \quad \triangleq \mathbb{P}\left(\inf _{0 \leq t \leq t_{1}} S_{1}(t) \geq H_{1}, S_{1}\left(t_{1}\right) \geq K_{1}, S_{2}\left(t_{1}\right) \leq K_{2}, \sup _{t_{1} \leq t \leq t_{2}} S_{2}(t) \leq H_{2}, S_{2}\left(t_{2}\right) \leq K_{3}, S_{3}\left(t_{3}\right) \leq K_{4}\right),
\end{aligned}
$$

(4)

$$
\begin{aligned}
& P_{\text {[up-and-down] }}\left(H_{1}, H_{2}, K_{1}, K_{2}, K_{3}, K_{4}, t_{1}, t_{2}, t_{3}\right) \\
& \quad \triangleq \mathbb{P}\left(\sup _{0 \leq t \leq t_{1}} S_{1}(t) \leq H_{1}, S_{1}\left(t_{1}\right) \leq K_{1}, S_{2}\left(t_{1}\right) \geq K_{2}, \inf _{t_{1} \leq t \leq t_{2}} S_{2}(t) \geq H_{2}, S_{2}\left(t_{2}\right) \geq K_{3}, S_{3}\left(t_{3}\right) \geq K_{4}\right) .
\end{aligned}
$$

Let us introduce the following new notations:

$$
k_{4}=\ln \left(\frac{K_{4}}{S_{3}\left(t_{0}\right)}\right), \quad \mu_{3}=\alpha_{3}-\frac{\sigma_{3}^{2}}{2} .
$$

The next proposition provides an exact formula for the four above-mentioned joint cumulative distribution functions.

Proposition 2.2. Let the real function $\Phi\left[b_{1}, b_{2}, b_{3}, b_{4} ; \theta_{1 \cdot 2}, \theta_{1 \cdot 3}, \theta_{1 \cdot 4}, \theta_{2 \cdot 3}, \theta_{3 \cdot 4}\right]$, where $\left\{b_{1}, b_{2}, b_{3}, b_{4}\right\}$ $\in \mathbb{R}^{4}$ and each of the real numbers $\theta_{1 \cdot 2}, \theta_{1 \cdot 3}, \theta_{1 \cdot 4}, \theta_{2 \cdot 3}, \theta_{3 \cdot 4}$ is included in $]-1,1[$, be defined as follows:

$$
\begin{aligned}
& \Phi\left[b_{1}, b_{2}, b_{3}, b_{4} ; \theta_{1 \cdot 2}, \theta_{1 \cdot 3}, \theta_{1 \cdot 4}, \theta_{2 \cdot 3}, \theta_{3 \cdot 4}\right] \\
& =\int_{x_{1}=-\infty}^{b_{1}} \int_{x_{2}=-\infty}^{b_{2}} \int_{x_{3}=-\infty}^{b_{3}} \frac{1}{\phi_{2 \mid 1} \phi_{3 \mid 2} \sqrt{8 \pi^{3}}} \exp \left(-\frac{x_{2}^{2}}{2}-\frac{\left(x_{1}-\theta_{1 \cdot 2} x_{2}\right)^{2}}{2 \phi_{2 \mid 1}^{2}}-\frac{\left(x_{3}-\theta_{2 \cdot 3} x_{2}\right)^{2}}{2 \phi_{3 \mid 2}^{2}}\right) \\
& \quad \times N\left[\frac{b_{4}-\theta_{1 \cdot 4} x_{1}-\theta_{3 \cdot 4 \mid 1}\left(\left(x_{3}-\theta_{1 \cdot 3} x_{1}\right) / \sqrt{1-\theta_{1 \cdot 3}^{2}}\right)}{\phi_{4 \mid 1 \cdot 3}}\right] d x_{3} d x_{2} d x_{1}
\end{aligned}
$$

where $N[\cdot]$ is the standard normal cumulative distribution function and the following definitions apply:

$$
\phi_{2 \mid 1}=\sqrt{1-\theta_{1 \cdot 2}^{2}}, \quad \phi_{3 \mid 2}=\sqrt{1-\theta_{2 \cdot 3}^{2}}, \quad \theta_{3 \cdot 4 \mid 1}=\frac{\theta_{3 \cdot 4}-\theta_{1 \cdot 3} \theta_{1 \cdot 4}}{\phi_{3 \mid 1}}, \quad \phi_{4 \mid 1 \cdot 3}=\sqrt{1-\theta_{1 \cdot 4}^{2}-\theta_{3 \cdot 4 \mid 1}^{2}} .
$$


Then, for the up-and-up and the down-and-down distributions, one has

$$
\begin{aligned}
& P_{[\cdot]}\left(H_{1}, H_{2}, K_{1}, K_{2}, K_{3}, K_{4}, t_{1}, t_{2}, t_{3}\right) \\
& =\Phi\left[\begin{array}{l}
\lambda\left(\frac{k_{1}-\mu_{1} t_{1}}{\sigma_{1} \sqrt{t_{1}}}\right), \lambda\left(\frac{k_{2}-\mu_{2} t_{1}}{\sigma_{2} \sqrt{t_{1}}}\right), \lambda\left(\frac{k_{3}-\mu_{2} t_{2}}{\sigma_{2} \sqrt{t_{2}}}\right), \lambda\left(\frac{k_{4}-\mu_{3} t_{3}}{\sigma_{3} \sqrt{t_{3}}}\right) ; \\
\theta_{1 \cdot 2}=\rho_{1 \cdot 2}, \theta_{1 \cdot 3}=\sqrt{\frac{t_{1}}{t_{2}}} \rho_{1 \cdot 2}, \theta_{1 \cdot 4}=\sqrt{\frac{t_{1}}{t_{3}}} \rho_{1 \cdot 3}, \theta_{2 \cdot 3}=\sqrt{\frac{t_{1}}{t_{2}}}, \theta_{3 \cdot 4}=\sqrt{\frac{t_{2}}{t_{3}}} \rho_{2 \cdot 3}
\end{array}\right] \\
& -\exp \left(\frac{2 \mu_{2} h_{2}}{\sigma_{2}^{2}}\right) \\
& \times \Phi\left[\begin{array}{l}
\lambda\left(\frac{k_{1}-\mu_{1} t_{1}}{\sigma_{1} \sqrt{t_{1}}}\right)+\rho_{1 \cdot 2} \frac{2 \mu_{2} t_{1}}{\sigma_{2} \sqrt{t_{1}}}, \lambda\left(\frac{k_{2}+\mu_{2} t_{1}}{\sigma_{2} \sqrt{t_{1}}}\right), \lambda\left(\frac{k_{3}-2 h_{2}-\mu_{2} t_{2}}{\sigma_{2} \sqrt{t_{2}}}\right), \\
\lambda\left(\frac{k_{4}-\mu_{3} t_{3}}{\sigma_{3} \sqrt{t_{3}}}-\frac{\theta_{3 \cdot 4}-\theta_{1 \cdot 3} \theta_{1 \cdot 4}}{\sqrt{1-\theta_{1 \cdot 3}^{2}}}\left(\frac{2 h_{2}}{\sigma_{2} \sqrt{t_{2}}}+\theta_{1 \cdot 3} \rho_{1 \cdot 2} \frac{2 \mu_{2} t_{1}}{\sigma_{2} \sqrt{t_{1}}}\right)+\theta_{1 \cdot 4} \rho_{1 \cdot 2} \frac{2 \mu_{2} t_{1}}{\sigma_{2} \sqrt{t_{1}}}\right) \\
\theta_{1 \cdot 2}=\rho_{1 \cdot 2}, \theta_{1 \cdot 3}=\sqrt{\frac{t_{1}}{t_{2}}} \rho_{1 \cdot 2}, \theta_{1 \cdot 4}=\sqrt{\frac{t_{1}}{t_{3}}} \rho_{1 \cdot 3}, \theta_{2 \cdot 3}=-\sqrt{\frac{t_{1}}{t_{2}}}, \theta_{3 \cdot 4}=\sqrt{\frac{t_{2}}{t_{3}}} \rho_{2 \cdot 3}
\end{array}\right] ; \\
& -\exp \left(\frac{2 \mu_{1} h_{1}}{\sigma_{1}^{2}}\right)
\end{aligned}
$$

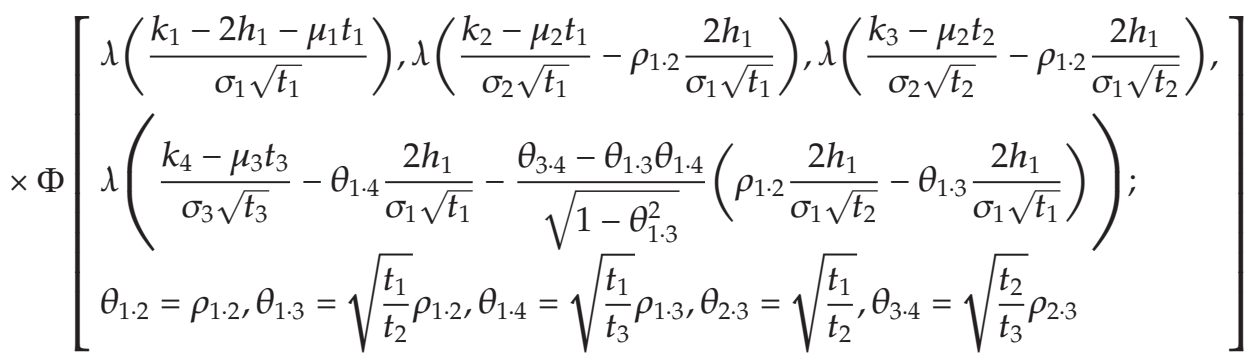

$$
\begin{aligned}
& +\exp \left(\left(\frac{2 \mu_{1}}{\sigma_{1}^{2}}-\frac{4 \mu_{2} \rho_{1 \cdot 2}}{\sigma_{1} \sigma_{2}}\right) h_{1}+\frac{2 \mu_{2} h_{2}}{\sigma_{2}^{2}}\right)
\end{aligned}
$$

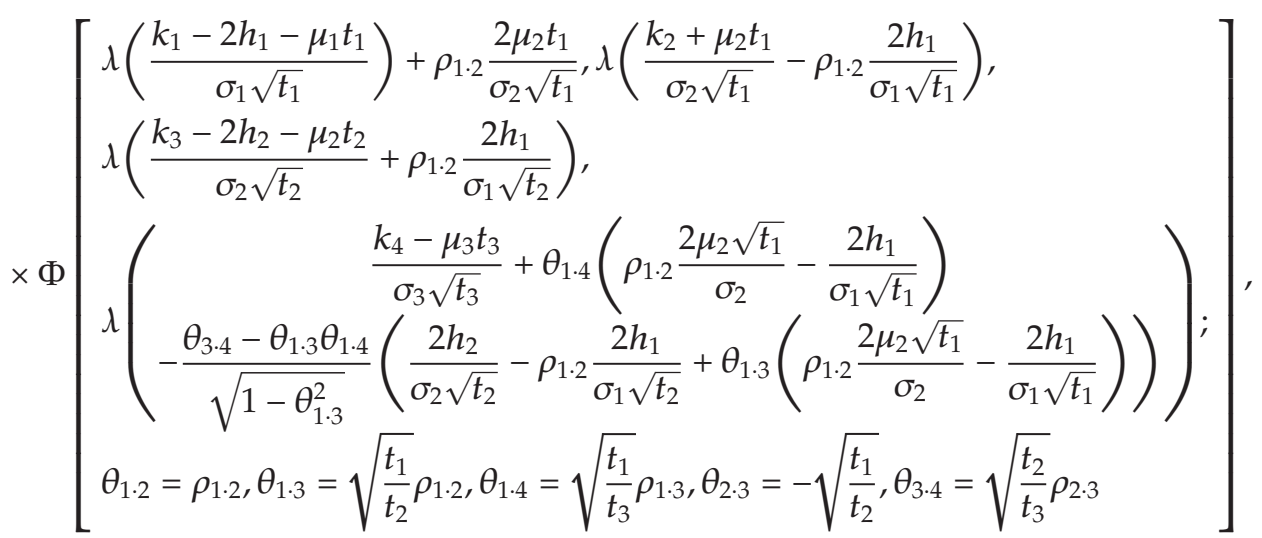


where

$$
\lambda=1 \text { if } P_{[\cdot]}\left(H_{1}, H_{2}, K_{1}, K_{2}, K_{3}, K_{4}, t_{1}, t_{2}, t_{3}\right)=P_{[\text {up-and-up }]}\left(H_{1}, H_{2}, K_{1}, K_{2}, K_{3}, K_{4}, t_{1}, t_{2}, t_{3}\right) \text {, }
$$

$\lambda=-1$ if $P_{[\cdot]}\left(H_{1}, H_{2}, K_{1}, K_{2}, K_{3}, K_{4}, t_{1}, t_{2}, t_{3}\right)=P_{\text {[down-and-down }]}\left(H_{1}, H_{2}, K_{1}, K_{2}, K_{3}, K_{4}, t_{1}, t_{2}, t_{3}\right)$.

And, for the up-and-down and the down-and-up distributions, one has

$$
\begin{aligned}
& P_{[.]}\left(H_{1}, H_{2}, K_{1}, K_{2}, K_{3}, K_{4}, t_{1}, t_{2}, t_{3}\right) \\
& =\Phi\left[\begin{array}{l}
\lambda\left(\frac{-k_{1}+\mu_{1} t_{1}}{\sigma_{1} \sqrt{t_{1}}}\right), \lambda\left(\frac{k_{2}-\mu_{2} t_{1}}{\sigma_{2} \sqrt{t_{1}}}\right), \lambda\left(\frac{k_{3}-\mu_{2} t_{2}}{\sigma_{2} \sqrt{t_{2}}}\right), \lambda\left(\frac{k_{4}-\mu_{3} t_{3}}{\sigma_{3} \sqrt{t_{3}}}\right) ; \\
\theta_{1 \cdot 2}=-\rho_{1 \cdot 2}, \theta_{1 \cdot 3}=-\sqrt{\frac{t_{1}}{t_{2}}} \rho_{1 \cdot 2}, \theta_{1 \cdot 4}=-\sqrt{\frac{t_{1}}{t_{3}}} \rho_{1 \cdot 3}, \theta_{2 \cdot 3}=\sqrt{\frac{t_{1}}{t_{2}}}, \theta_{3 \cdot 4}=\sqrt{\frac{t_{2}}{t_{3}}} \rho_{2 \cdot 3}
\end{array}\right] \\
& -\exp \left(\frac{2 \mu_{2} h_{2}}{\sigma_{2}^{2}}\right) \\
& \times \Phi\left[\begin{array}{l}
\lambda\left(\frac{-k_{1}+\mu_{1} t_{1}}{\sigma_{1} \sqrt{t_{1}}}\right)+\rho_{1 \cdot 2} \frac{2 \mu_{2} t_{1}}{\sigma_{2} \sqrt{t_{1}}}, \lambda\left(\frac{k_{2}+\mu_{2} t_{1}}{\sigma_{2} \sqrt{t_{1}}}\right), \lambda\left(\frac{k_{3}-2 h_{2}-\mu_{2} t_{2}}{\sigma_{2} \sqrt{t_{2}}}\right), \\
\lambda\left(\frac{k_{4}-\mu_{3} t_{3}}{\sigma_{3} \sqrt{t_{3}}}-\frac{\theta_{3 \cdot 4}-\theta_{1 \cdot 3} \theta_{1 \cdot 4}}{\sqrt{1-\theta_{1 \cdot 3}^{2}}}\left(\frac{2 h_{2}}{\sigma_{2} \sqrt{t_{2}}}+\theta_{1 \cdot 3} \rho_{1 \cdot 2} \frac{2 \mu_{2} \sqrt{t_{1}}}{\sigma_{2}}\right)+\theta_{1 \cdot 4} \rho_{1 \cdot 2} \frac{2 \mu_{2} \sqrt{t_{1}}}{\sigma_{2}}\right) \\
\theta_{1 \cdot 2}=-\rho_{1 \cdot 2}, \theta_{1 \cdot 3}=\sqrt{\frac{t_{1}}{t_{2}}} \rho_{1 \cdot 2}, \theta_{1 \cdot 4}=-\sqrt{\frac{t_{1}}{t_{3}}} \rho_{1 \cdot 3}, \theta_{2 \cdot 3}=-\sqrt{\frac{t_{1}}{t_{2}}}, \theta_{3 \cdot 4}=\sqrt{\frac{t_{2}}{t_{3}}} \rho_{2 \cdot 3}
\end{array}\right] \\
& -\exp \left(\frac{2 \mu_{1} h_{1}}{\sigma_{1}^{2}}\right)
\end{aligned}
$$

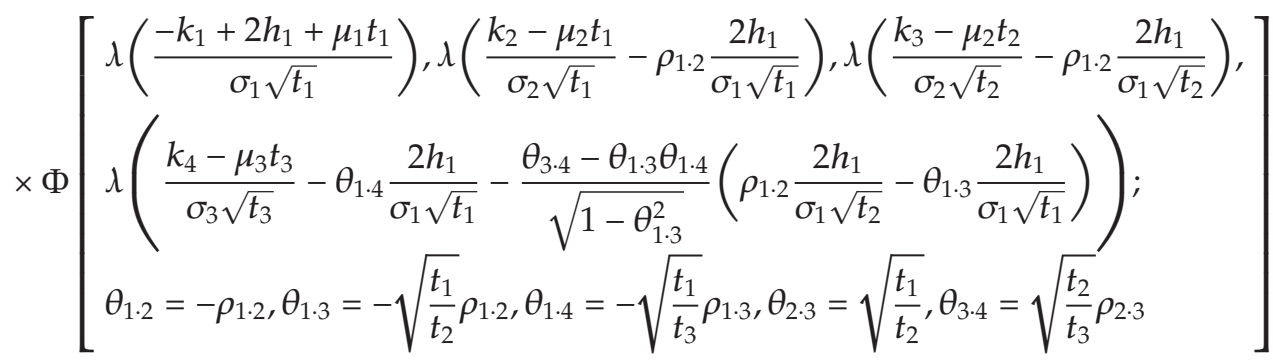

$$
\begin{aligned}
& +\exp \left(\left(\frac{2 \mu_{1}}{\sigma_{1}^{2}}-\frac{4 \mu_{2} \rho_{1 \cdot 2}}{\sigma_{1} \sigma_{2}}\right) h_{1}+\frac{2 \mu_{2} h_{2}}{\sigma_{2}^{2}}\right)
\end{aligned}
$$




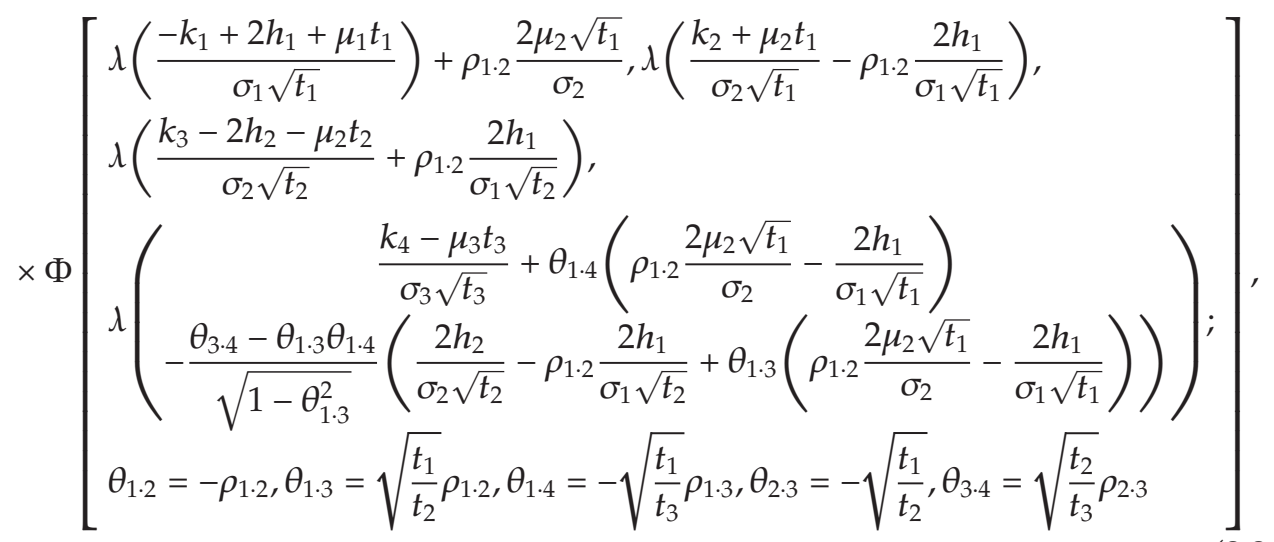

where

$$
\begin{aligned}
& \lambda=1 \quad \text { if } P_{[\cdot]}\left(H_{1}, H_{2}, K_{1}, K_{2}, K_{3}, K_{4}, t_{1}, t_{2}, t_{3}\right)=P_{[\text {down-and-up }]}\left(H_{1}, H_{2}, K_{1}, K_{2}, K_{3}, K_{4}, t_{1}, t_{2}, t_{3}\right), \\
& \lambda=-1 \quad \text { if } P_{[\cdot]}\left(H_{1}, H_{2}, K_{1}, K_{2}, K_{3}, K_{4}, t_{1}, t_{2}, t_{3}\right)=P_{[\text {up-and-down }]}\left(H_{1}, H_{2}, K_{1}, K_{2}, K_{3}, K_{4}, t_{1}, t_{2}, t_{3}\right) .
\end{aligned}
$$

\section{Corollary of Proposition 2.2}

The four following cumulative distribution functions, that will be useful in Section 3, are deduced from Proposition 2.2:

(1)

$$
\begin{gathered}
\mathbb{P}\left(\sup _{0 \leq t \leq t_{1}} S_{1}(t) \leq H_{1}, S_{1}\left(t_{1}\right) \leq K_{1}, S_{2}\left(t_{1}\right) \leq K_{2}, \sup _{t_{1} \leq t \leq t_{2}} S_{2}(t) \leq H_{2}, S_{2}\left(t_{2}\right) \leq K_{3}, S_{3}\left(t_{3}\right) \geq K_{4}\right) \\
=P_{\text {[up-and-up] }}\left(H_{1}, H_{2}, K_{1}, K_{2}, K_{3}, \infty\right)-P_{\text {[up-and-up] }}\left(H_{1}, H_{2}, K_{1}, K_{2}, K_{3}, K_{4}\right),
\end{gathered}
$$

(2)

$$
\begin{gathered}
\mathbb{P}\left(\inf _{0 \leq t \leq t_{1}} S_{1}(t) \geq H_{1}, S_{1}\left(t_{1}\right) \geq K_{1}, S_{2}\left(t_{1}\right) \geq K_{2}, \inf _{t_{1} \leq t \leq t_{2}} S_{2}(t) \geq H_{2}, S_{2}\left(t_{2}\right) \geq K_{3}, S_{3}\left(t_{3}\right) \leq K_{4}\right) \\
\quad=P_{\text {[down-and-down] }}\left(H_{1}, H_{2}, K_{1}, K_{2}, K_{3},-\infty\right)-P_{\text {[down-and-down] }}\left(H_{1}, H_{2}, K_{1}, K_{2}, K_{3}, K_{4}\right),
\end{gathered}
$$


(3)

$$
\begin{gathered}
\mathbb{P}\left(\inf _{0 \leq t \leq t_{1}} S_{1}(t) \geq H_{1}, S_{1}\left(t_{1}\right) \geq K_{1}, S_{2}\left(t_{1}\right) \leq K_{2}, \sup _{t_{1} \leq t \leq t_{2}} S_{2}(t) \leq H_{2}, S_{2}\left(t_{2}\right) \leq K_{3}, S_{3}\left(t_{3}\right) \geq K_{4}\right) \\
=P_{\text {[down-and-up] }}\left(H_{1}, H_{2}, K_{1}, K_{2}, K_{3}, \infty\right)-P_{\text {[down-and-up] }}\left(H_{1}, H_{2}, K_{1}, K_{2}, K_{3}, K_{4}\right),
\end{gathered}
$$

$$
\begin{gathered}
\mathbb{P}\left(\sup _{0 \leq t \leq t_{1}} S_{1}(t) \leq H_{1}, S_{1}\left(t_{1}\right) \leq K_{1}, S_{2}\left(t_{1}\right) \geq K_{2}, \inf _{t_{1} \leq t \leq t_{2}} S_{2}(t) \geq H_{2}, S_{2}\left(t_{2}\right) \geq K_{3}, S_{3}\left(t_{3}\right) \leq K_{4}\right) \\
=P_{\text {[up-and-down] }}\left(H_{1}, H_{2}, K_{1}, K_{2}, K_{3},-\infty\right)-P_{\text {[up-and-down] }}\left(H_{1}, H_{2}, K_{1}, K_{2}, K_{3}, K_{4}\right) .
\end{gathered}
$$

Proof of Proposition 2.2 is provided in the Appendices A, B, and C.

Practical use of Proposition 2.2 and its corollary in engineering applications depend on the accuracy and the efficiency with which the function $\Phi$ can be numerically implemented. This question is dealt with in Section 3.

\section{Applications and Numerical Implementation}

This Section deals with applications of the results provided in Section 1 in financial engineering. Indeed, Propositions 2.1 and 2.2 can be used as the building blocks for the valuation and the risk management of a large number of option contracts. The main class of instruments under consideration will be barrier options. In their standard form, the latter are contracts whereby the holder is entitled (but not obligated) to buy (call option) or sell (put option) an asset at a prespecified future date (the option expiry) at a prespecified price (the strike price), on condition that the asset price has not (knock-out type) or has (knock-in type) crossed a specific upward level (called the up-barrier) or downward level (called the down-barrier) at any given time from valuation date to expiry. In standard option pricing theory, asset prices are modelled as geometric Brownian motions, so that boundary crossing distributions such as those provided in Section 1 are of immediate use in financial engineering.

It must be emphasized that the market for barrier options is huge. They are the most actively traded class of nonstandard options (usually referred to as exotic options). Many variations on the standard barrier option payoff have been designed to match investors' demand more closely. One of them is the partial-time barrier option, which specifies that the barrier level is monitored during only a fraction of the option lifespan. The basic contracts were studied by Heynen and Kat [11], and more general contracts were valued by Armstrong [12] and Guillaume [13]. Another variation is the step barrier option (Guillaume [6,9]), whereby the barrier evolves as a step function of time. It is also common to encounter outside barrier options, whose payoff is a function of two asset prices: one of them is compared with the strike price at expiry to determine the moneyness of the option, while the other one is 
monitored up until expiry to check whether the barrier level has been crossed. Outside barrier options were originally valued by Heynen and Kat [14], and they were further studied by Kwok et al. [15] and Wong and Kwok [16].

These different features: partial time barrier, step barrier, and outside barrier, often combine to allow for increased flexibility. But then, performing analytical valuation becomes more and more involved and practitioners have to turn to numerical methods that are slow and relatively inaccurate. This is when the results provided in Section 1 become valuable. Indeed, they enable to price a large number of barrier option contracts sharing the three above-mentioned innovative features (partial time, step, and outside barrier), that is, options based on two or even three assets that may knock out depending on whether the underlying assets move within a sequence of prespecified ranges of prices over all or part of the option lifespan. More precisely, Proposition 2.1 and its corollary are the building blocks for the valuation of sequential two-asset knock-out calls and puts whose four pay-off structures are defined as follows:

(1) $\left(K-S_{t_{2}}^{(2)}\right)^{+} \mathbb{I}_{\left\{\sup _{0 \leq \leqslant \leq t_{1}} S_{t}^{(1)}<H_{1}, \sup _{t_{1} \leq \leq \leq t_{2}} S_{t}^{(2)}<H_{2}\right\}}$ for a two-asset up-and-up knock-out put (substitute $\left(S_{t_{2}}^{(2)}-K\right)^{+}$for $\left(K-S_{t_{2}}^{(2)}\right)^{+}$for an up-and-up knock-out call),

(2) $\pm\left(S_{t_{2}}^{(2)}-K\right)^{+} \mathbb{I}_{\left\{\text {inf }_{0 \leq \leq \leq t} S_{1}^{(1)}>H_{1}, \text { inf }_{t_{1} \leq \leq \leq t_{2}} S_{t}^{(2)}>H_{2}\right\}}$ for a two-asset down-and-down knock-out call or put,

(3) $\pm\left(S_{t_{2}}^{(2)}-K\right)^{+} \mathbb{I}_{\left\{\sup _{0 \leq \leq \leq \leq t} S_{t}^{(1)}<H_{1}, \inf _{t_{1} \leq \leq \leq t} S_{t}^{(2)}>H_{2}\right\}}$ for a two-asset up-and-down knock-out call or put,

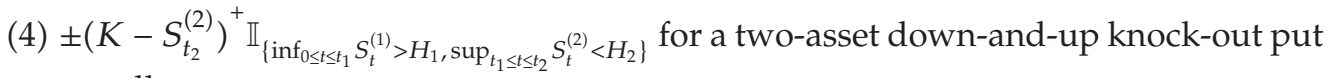
or call,

where:

(i) $\mathbb{I}_{\{\}}$is the indicator function,

(ii) $\left\{S_{1}(t), t \geq 0\right\}$ and $\left\{S_{2}(t), t \geq 0\right\}$ are the price processes of two risky assets,

(iii) $K$ is the strike price and $t_{2}$ is the option expiry,

(iv) $H_{1}$ is a knock-out barrier monitored with respect to $\left\{S_{1}(t), t \geq 0\right\}$ over the time interval $\left[t_{0}, t_{1}\right]$,

(v) $H_{2}$ is a knock-out barrier monitored with respect to $\left\{S_{2}(t), t \geq 0\right\}$ over the time interval $\left[t_{1}, t_{2}\right]$.

The no-arbitrage price of these eight different option contracts is now provided by the following proposition.

Proposition 3.1. Let two geometric Brownian motions $\left\{S_{1}(t), t \geq 0\right\}$ and $\left\{S_{2}(t), t \geq 0\right\}$ be the price processes of two risky assets with constant volatilities $\sigma_{1}$ and $\sigma_{2}$, respectively, and constant correlation coefficient $\rho_{1 \cdot 2}$. Let $\delta_{1}$ and $\delta_{2}$ be two constant pay-out rates on assets $S_{1}$ and $S_{2}$, respectively, and $r$ be the constant risk-free interest rate. Let $v_{1}=r-\delta_{1}+\sigma_{1} \sigma_{2} \rho_{1 \cdot 2}$ and $v_{2}=r-\delta_{2}+\sigma_{2}^{2}$.

The value, at time $t_{0}=0$, of a two-asset sequential knock-out option with strike price $K$, with knock-out barriers $H_{1}$ and $H_{2}$ monitored with respect to $S_{1}$ over the time interval $\left[t_{0}, t_{1}\right]$ and $S_{2}$ over the time interval $\left[t_{1}, t_{2}\right]$, respectively, and with maturity $t_{2}, t_{2} \geq t_{1} \geq t_{0}$, is given by

$$
V\left(S_{1}\left(t_{0}\right), S_{2}\left(t_{0}\right), t_{1}, t_{2}, K, H_{1}, H_{2}\right)= \pm\left[K \exp \left(-r t_{2}\right) P_{1}-S_{2}\left(t_{0}\right) \exp \left(-\delta_{2} t_{2}\right) P_{2}\right]
$$


where the following specifications hold.

(1) If the option is an up-and-up put,

$\pm=1$, and $P_{1}$ is obtained by substituting $\alpha_{1}=r-\delta_{1}$ and $\alpha_{2}=r-\delta_{2}$ in $P_{[u p-a n d-u p]}\left(H_{1}, H_{2}, H_{1}, H_{2}, K, t_{1}, t_{2}\right)$ in Proposition 2.1, and $P_{2}$ is obtained by substituting $\alpha_{1}=v_{1}$ and $\alpha_{2}=v_{2}$ in $P_{\text {[up-and-up }]}\left(H_{1}, H_{2}, H_{1}, H_{2}, K, t_{1}, t_{2}\right)$ in Proposition 2.1.

(2) If the option is a down-and-down call,

$\pm=-1$, and $P_{1}$ is obtained by substituting $\alpha_{1}=r-\delta_{1}$ and $\alpha_{2}=r-\delta_{2}$ in $P_{\text {[down-and-down }]}\left(H_{1}, H_{2}, H_{1}, H_{2}, K, t_{1}, t_{2}\right)$ in Proposition 2.1, and $P_{2}$ is obtained by substituting $\alpha_{1}=v_{1}$ and $\alpha_{2}=v_{2}$ in $P_{\text {[down-and-down }]}\left(H_{1}, H_{2}, H_{1}, H_{2}, K, t_{1}, t_{2}\right)$ in Proposition 2.1.

(3) If the option is an up-and-down call,

$\pm=-1$, and $P_{1}$ is obtained by substituting $\alpha_{1}=r-\delta_{1}$ and $\alpha_{2}=r-$ $\delta_{2}$ in $P_{\text {[up-and-down] }}\left(H_{1}, H_{2}, H_{1}, H_{2}, K, t_{1}, t_{2}\right)$ in Proposition 2.1 and $P_{2}$ is obtained by substituting $\alpha_{1}=v_{1}$ and $\alpha_{2}=v_{2}$ in $P_{[\text {[up-and-down }]}\left(H_{1}, H_{2}, H_{1}, H_{2}, K, t_{1}, t_{2}\right)$ in Proposition 2.1.

(4) If the option is a down-and-up put,

$\pm=1$, and $P_{1}$ is obtained by substituting $\alpha_{1}=r-\delta_{1}$ and $\alpha_{2}=r-$ $\delta_{2}$ in $P_{\text {[down-and-up] }}\left(H_{1}, H_{2}, H_{1}, H_{2}, K, t_{1}, t_{2}\right)$ in Proposition 2.1 and $P_{2}$ is obtained by substituting $\alpha_{1}=v_{1}$ and $\alpha_{2}=v_{2}$ in $P_{\text {[down-and-up] }}\left(H_{1}, H_{2}, H_{1}, H_{2}, K, t_{1}, t_{2}\right)$ in Proposition 2.1.

By using Corollary of Proposition 2.1, one can value up-and-up and down-and-up call options, as well as down-and-down and up-and-down put options.

A sketch of proof of Proposition 3.1 is provided in the Appendices A, B, and C.

Just as Proposition 2.1 enables to value two-asset knock-out calls and puts, Proposition 2.2 can be used to value three-asset knock-out calls and puts. More specifically, if $\left\{S_{3}(t), t \geq 0\right\}$ is the price process of a third risky asset and $t_{3}$ is the new option expiry, with $t_{3} \geq t_{2} \geq t_{1} \geq t_{0}$, then the four following pay-off structures can be analytically tackled:

(1) $\pm\left(K-S_{t_{3}}^{(3)}\right)^{+} \mathbb{I}_{\left\{\sup _{0 \leq \leq \leq \leq t} S_{1}^{(1)}<H_{1}, \sup _{t_{1} \leq \leq \leq t_{2}} S_{t}^{(2)}<H_{2}\right\}}$ for a three-asset up-and-up knock-out put or call,

(2) $\pm\left(S_{t_{3}}^{(3)}-K\right)^{+} \mathbb{I}_{\left\{\text {inf }_{0 \leq \leq \leq t_{1}} S_{t}^{(1)}>H_{1}, \text { inf }_{t_{1} \leq \leq \leq t_{2}} S_{t}^{(2)}>H_{2}\right\}}$ for a three-asset down-and-down knock-out call or put,

(3) $\pm\left(S_{t_{3}}^{(3)}-K\right)^{+} \mathbb{I}_{\left\{\sup _{0 \leq \leq \leq t} S_{1}^{(1)}<H_{1}, \inf _{t_{1} \leq \leq \leq t_{2}} S_{t}^{(2)}>H_{2}\right\}}$ for a three-asset up-and-down knock-out call or put,

(4) $\pm\left(K-S_{t_{3}}^{(3)}\right)^{+} \mathbb{I}_{\left\{\text {inf }_{0 \leq \leq \leq t_{1}} S_{t}^{(1)}>H_{1}, \sup _{t_{1} \leq \leqslant \leq t_{2}} S_{t}^{(2)}<H_{2}\right\}}$ for a three-asset down-and-up knock-out put or call.

The no-arbitrage prices of the eight types of option under consideration are now provided by the following proposition.

Proposition 3.2. Let three geometric Brownian motions $\left\{S_{1}(t), t \geq 0\right\},\left\{S_{2}(t), t \geq 0\right\}$, and $\left\{S_{3}(t), t \geq\right.$ $0\}$ be the price processes of three risky assets with constant volatilities $\sigma_{1}, \sigma_{2}$, and $\sigma_{3}$, respectively, and 
constant pairwise correlation coefficients $\rho_{a \cdot b},(a, b) \in\{1,2,3\}$, at any fixed time $t \geq 0$. Let $\delta_{1}, \delta_{2}$, and $\delta_{3}$ be three constant pay-out rates on assets $S_{1}, S_{2}$, and $S_{3}$, respectively, and $r$ be the constant risk-free interest rate. Let $v_{1}=r-\delta_{1}+\sigma_{1} \sigma_{3} \rho_{1 \cdot 3}, v_{2}=r-\delta_{2}+\sigma_{2} \sigma_{3} \rho_{2 \cdot 3}$, and $v_{3}=r-\delta_{3}+\sigma_{3}^{2}$.

The value, at time $t_{0}=0$, of a three-asset sequential knock-out option with strike price $K$, with knock-out barriers $H_{1}$ and $H_{2}$ monitored with respect to $S_{1}$ over the time interval $\left[t_{0}, t_{1}\right]$ and $S_{2}$ over the time interval $\left[t_{1}, t_{2}\right]$, respectively, with maturity $t_{3}, t_{3} \geq t_{2} \geq t_{1} \geq t_{0}$, is given by

$$
V\left(S_{1}\left(t_{0}\right), S_{2}\left(t_{0}\right), S_{3}\left(t_{0}\right), t_{1}, t_{2}, t_{3}, K, H_{1}, H_{2}\right)= \pm\left[K \exp \left(-r t_{3}\right) P_{1}-S_{3}\left(t_{0}\right) \exp \left(-\delta_{3} t_{3}\right) P_{2}\right]
$$

where the following specifications hold.

(1) If the option is an up-and-up put,

$\pm=1$, and $P_{1}$ is obtained by substituting $\alpha_{1}=r-\delta_{1}, \alpha_{2}=r-\delta_{2}$ and $\alpha_{3}=r-\delta_{3}$ in $P_{\text {[up-and-up] }}\left(H_{1}, H_{2}, H_{1}, H_{2}, H_{2}, K, t_{1}, t_{2}, t_{3}\right)$ in Proposition 2.2, and $P_{2}$ is obtained by substituting $\alpha_{1}=v_{1}, \alpha_{2}=v_{2}$ and $\alpha_{3}=v_{3}$ in $P_{[u p-a n d-u p]}\left(H_{1}, H_{2}, H_{1}, H_{2}, H_{2}, K, t_{1}, t_{2}, t_{3}\right)$ in Proposition 2.2.

(2) If the option is a down-and-down call,

$\pm=-1$, and $P_{1}$ is obtained by substituting $\alpha_{1}=r-\delta_{1}, \alpha_{2}=r-\delta_{2}$ and $\alpha_{3}=r-\delta_{3}$ in $P_{\text {[down-and-down] }}\left(H_{1}, H_{2}, H_{1}, H_{2}, H_{2}, K, t_{1}, t_{2}, t_{3}\right)$ in Proposition 2.2, and $P_{2}$ is obtained by substituting $\alpha_{1}=v_{1}, \alpha_{2}=v_{2}$ and $\alpha_{3}=v_{3}$ in $P_{\text {[down-and-down }]}\left(H_{1}, H_{2}, H_{1}, H_{2}, H_{2}, K, t_{1}, t_{2}, t_{3}\right)$ in Proposition 2.2.

(3) If the option is an up-and-down call,

$\pm=-1$, and $P_{1}$ is obtained by substituting $\alpha_{1}=r-\delta_{1}, \alpha_{2}=r-\delta_{2}$ and $\alpha_{3}=r-\delta_{3}$ in $P_{\text {[up-and-down }]}\left(H_{1}, H_{2}, H_{1}, H_{2}, H_{2}, K, t_{1}, t_{2}, t_{3}\right)$ in Proposition 2.2 and $P_{2}$ is obtained by substituting $\alpha_{1}=v_{1}, \alpha_{2}=v_{2}$ and $\alpha_{3}=v_{3}$ in $P_{\text {[up-and-down] }}\left(H_{1}, H_{2}, H_{1}, H_{2}, H_{2}, K, t_{1}, t_{2}, t_{3}\right)$ in Proposition 2.2.

(4) If the option is a down-and-up put, $\pm=1$, and $P_{1}$ is obtained by substituting $\alpha_{1}=r-\delta_{1}, \alpha_{2}=r-\delta_{2}$ and $\alpha_{3}=r-\delta_{3}$ in $P_{[\text {down-and-up }]}\left(H_{1}, H_{2}, H_{1}, H_{2}, H_{2}, K, t_{1}, t_{2}, t_{3}\right)$ in Proposition 2.2 and $P_{2}$ is obtained by substituting $\alpha_{1}=v_{1}, \alpha_{2}=v_{2}$ and $\alpha_{3}=v_{3}$ in $P_{\text {[down-and-up] }}\left(H_{1}, H_{2}, H_{1}, H_{2}, H_{2}, K, t_{1}, t_{2}, t_{3}\right)$ in Proposition 2.2.

By using Corollary of Proposition 2.2, one can value up-and-up and down-and-up call options, as well as down-and-down and up-and-down put options.

A sketch of proof of Proposition 3.2 is provided in the Appendices A, B, and C. conditions:

It is easy to notice that Proposition 3.2 nests Proposition 3.1 under the following three

(1) $v_{1}=r-\delta_{1}+\sigma_{1} \sigma_{2} \rho_{1 \cdot 2}$ and $v_{2}=r-\delta_{2}+\sigma_{2}^{2}$,

(2) take $P_{[\cdot]}\left(H_{1}, H_{2}, H_{1}, H_{2}, K, U, t_{1}, t_{2}, t_{3}\right)$ with $U \rightarrow \infty$ if you are dealing with a put and with $U \rightarrow-\infty$ if you are dealing with a call,

(3) compute $\pm\left[K \exp \left(-r t_{2}\right) P_{1}-S_{2}\left(t_{0}\right) \exp \left(-\delta_{2} t_{2}\right) P_{2}\right]$.

It is useful, though, to state Proposition 3.1, as it uses more standard functions that can be numerically computed with known high accuracy and thus can serve as a benchmark to test the reliability of the numerical implementation of Proposition 3.2. 
It can also be noticed that Proposition 3.2 enables to value sequential two-asset knockout options having the property that the moneyness of the option at expiry is tested with respect to asset $S_{1}$ instead of asset $S_{2}$ as in Proposition 3.1. This is achieved by implementing Proposition 3.2 with the following specifications:

(1) $v_{1}=r-\delta_{1}+\sigma_{1}^{2}$ and $v_{2}=r-\delta_{2}+\sigma_{1} \sigma_{2} \rho_{1 \cdot 2}$,

(2) $t_{3} \rightarrow t_{2}, \sigma_{3} \rightarrow \sigma_{1}, \rho_{1 \cdot 3} \rightarrow 1$ and $\rho_{2 \cdot 3} \rightarrow \rho_{1 \cdot 2}$

(3) compute $\pm\left[K \exp \left(-r t_{2}\right) P_{1}-S_{1}\left(t_{0}\right) \exp \left(-\delta_{1} t_{1}\right) P_{2}\right]$.

Similarly, Proposition 3.2 can be used to value sequential two-asset knock-out options with expiry $t_{3}$, so that, overall, this formula alone suffices to compute 40 different types of sequential multiasset knock-out options.

Proposition 3.2 implies the numerical implementation of Proposition 2.2. The function $\Phi$ in Proposition 2.2 involves three-dimensional numerical integration. Given the fact that the integrand is very smooth, a classical Gauss-Legendre quadrature can be used, along with a simple adaptive rule increasing the number of points in case the required accuracy is not reached after one iteration, starting with an 8-point quadrature. To test the efficiency and the accuracy of this numerical implementation, four series of tests have been implemented. In the first three categories of tests, the option parameters are specified so that the option can be valued by a known analytical formula involving numerical integration of lower dimension. More specifically, tests of category 1 reduce a three-asset up-and-up knock-out put to a two-asset up-and-up knock-out put by letting $K_{4} \rightarrow \infty$ in $P_{\text {[up-and-up] }}\left(H_{1}, H_{2}, K_{1}, K_{2}, K_{3}, K_{4}, t_{1}, t_{2}, t_{3}\right)$; the benchmark is then Proposition 3.1, that involves the computation of trivariate normal cumulative distribution functions. The latter can be obtained with high precision by Genz's algorithm (Genz [10]), as mentioned earlier.

Tests of category 2 reduce the three-asset up-and-up knock-out put to an outside knock-out put by letting $K_{1}=H_{1}, H_{2} \rightarrow \infty, K_{3} \rightarrow \infty, K_{4} \rightarrow \infty$, and $t_{3} \rightarrow t_{2} \rightarrow t_{1}$ in $P_{\text {[up-and-up] }}\left(H_{1}, H_{2}, K_{1}, K_{2}, K_{3}, K_{4}, t_{1}, t_{2}, t_{3}\right)$; the benchmark, then, is the formula for an outside barrier option as given by Heynen and Kat [14], that involves the computation of bivariate normal cumulative distribution functions. Again, the latter can be obtained with high precision using Genz [10].

Tests of category 3 reduce the three-asset up-and-up knock-out put to a single asset up-and-out put by letting $H_{2} \rightarrow \infty, K_{2}=K_{1}, K_{3} \rightarrow \infty, K_{4} \rightarrow \infty, t_{3} \rightarrow t_{2} \rightarrow t_{1}, \sigma_{2}=\sigma_{1}$ and $\rho_{1.2} \rightarrow 1$ in $P_{\text {[up-and-up] }}\left(H_{1}, H_{2}, K_{1}, K_{2}, K_{3}, K_{4}, t_{1}, t_{2}, t_{3}\right)$; the benchmark, then, is the formula for a basic barrier option as given, for example, by Haug [17], that involves the computation of univariate normal cumulative distribution functions.

In the last set of tests, the three-asset up-and-up knock-out put cannot be valued as a simpler contract, so that no reduction of the dimension of numerical integration is possible. Our goal, then, is to find a robust numerical valuation method and study its convergence pattern with the results obtained by implementing Proposition 3.2. Neither using trees nor numerically solving a partial differential equation stands as the most suitable methods in this case, in view of the double difficulty of having three correlated diffusion processes and path-dependent features in the option payoff. On another hand, crude Monte Carlo simulation would be very poor both in terms of accuracy and efficiency, due to the required time discretization. That is why the most suitable numerical method in this case is probably a Brownian Bridge Monte Carlo approximation (Glasserman [18]), as it involves only 6 random draws of the asset prices for each simulation and avoids a discretization bias. This is indeed the method commonly used by market practitioners when they are faced with a pricing problem involving both multiasset and path-dependent features. 
For each category of tests, a total of 10,000 different option prices have been computed with randomly drawn volatility, correlation, and expiry parameters. Let us now sum up the main findings of these numerical experiments. First, the average computational time when applying Proposition 3.2 on an ordinary personal computer (P7350 2GHz) is 0.6 seconds, which is fast. The longest recorded computational time was 1.4 second. Speed could obviously be greatly increased by involving more hardware resources. In absolute terms, Proposition 3.2 can thus be considered as an efficient way of computing option prices. In relative terms, Proposition 3.2 is even extremely efficient. Indeed, 10,000,000 simulations are required to obtain a modest $10^{-4}$ digits convergence of the Brownian Bridge Monte Carlo approximation with Proposition 3.2, which amounts to a computational time of over 4 minutes! Such slowness becomes a serious issue in real-time trading or when large portfolios of options are valued. As far as accuracy is concerned, the results obtained using Proposition 3.2 always matched to at least $10^{-8}$ digits the analytical benchmarks in almost all the numerical experiments conducted. The very rare exceptions were observed when the absolute value of one of the correlation coefficients was above $99.7 \%$. It must be noticed that in this couple of cases, the correlation matrix was found to be almost singular. Besides, risky assets with such levels of correlation are never encountered in the markets, so that it does not seem worthwhile to us to search for more complicated integration rules that would be better suited to these negligible subregions of integration. For all practical purposes, the numerical accuracy of our simple implementation of Proposition 3.2 can thus be deemed as very satisfactory. The results did not vary significantly between tests of categories 1,2, and 3, which means that an increase in the number of option parameters that are given limit values does not entail instability in the quadrature rule.

To close this section, it is worth mentioning that the results provided in Section 1 can also apply to other popular derivatives contracts. Among them is the look-back option, which allows investors to sell at the highest and buy at the lowest over a time interval, thus optimizing market exit and market entry on a given asset. In its basic form, a fixed strike look-back call pays off $\left(\sup _{0 \leq t \leq T} S(t)-K\right)^{+}$at expiry $T$. Many variations on this standard structure have been designed. One of them is the double look-back call, written on two assets $S_{1}$ and $S_{2}$, which provides investors with the following payoff at expiry $T:\left(a_{1} \sup _{0 \leq t \leq T} S_{1}(t)-a_{2} \sup _{0 \leq t \leq T} S_{2}(t)-K\right)^{+}$, where $a_{1}$ and $a_{2}$ are two positive weights. The valuation of this contract has been studied by $\mathrm{He}$ et al. [8]. A sequential double look-back call is a variation on the double look-back call. Its payoff at expiry $t_{2}$ writes $\left(a_{1} \sup _{0 \leq t \leq t_{1}} S_{1}(t)-a_{2} \sup _{t_{1} \leq t \leq t_{2}} S_{2}(t)-K\right)^{+}$. Drawing on Proposition 2.1, the no-arbitrage price of this option is equal to

$$
\begin{array}{r}
\exp \left(-r t_{2}\right) \int_{h_{1}=0}^{\infty} \int_{h_{2}=-\infty}^{\infty}\left(a_{1} S_{1}\left(t_{0}\right) \exp \left(h_{1}\right)-a_{2} S_{2}\left(t_{0}\right) \exp \left(h_{2}\right)-K\right)^{+} \\
\frac{\partial^{2} P_{\text {[up-and-up] }}\left(H_{1}, H_{2}, H_{1}, H_{2}, H_{2}\right)}{\partial h_{1} \partial h_{2}} d h_{2} d h_{1},
\end{array}
$$

where $\alpha_{1}=r-\delta_{1}$ and $\alpha_{2}=r-\delta_{2}$ in Proposition 2.1.

The partial derivative in (3.3) can be determined by analytical differentiation of $P_{\text {[up-and-up] }}\left(H_{1}, H_{2}, H_{1}, H_{2}, H_{2}\right)$, but this is relatively cumbersome. Given the simplicity of the computation of Proposition 2.1, it is faster and easier to compute those partial derivatives numerically. One can notice that the valuation of sequential double look-back options is only semianalytical since it still requires numerical double integration. For a given degree 
of the quadrature rule, it will not be as accurate as the valuation of two-asset and threeasset sequential knock-out options because of the inaccuracies arising from the numerical differentiation of Proposition 2.1.

\section{Conclusion}

This paper provides new explicit solutions for some sequential boundary crossing problems in a multidimensional geometric Brownian motion framework. Among their various possible applications, they form the basis for accurate and fast valuation of a large number of option contracts traded in the markets. The methods presented here could be used to solve higher-dimensional problems, especially the introduction of a third boundary monitored with respect to the third process in the model. However, the resulting formulae would become quite cumbersome and, more importantly, the quality of the numerical integration involved should have to be compared with a Brownian Bridge Monte Carlo approximation. Another possible extension would be to study the analytical tractability of introducing curved boundaries, but it should be pointed out that this form of boundary is rarely encountered in the options markets.

\section{Appendices}

\section{A. Proof of Proposition 2.1}

Proof is outlined for $P_{\text {[up-and-up] }}\left(H_{1}, H_{2}, K_{1}, K_{2}, K_{3}, t_{1}, t_{2}\right)$ only. The other distributions are obtained in a similar manner.

The first step is to take the logarithms of $S_{1}\left(t_{1}\right), S_{2}\left(t_{1}\right)$, and $S_{2}\left(t_{2}\right)$, in order to move from lognormal random variables to normal random variables. The sought cumulative distribution becomes

$$
\mathbb{P}\left(\sup _{0 \leq t \leq t_{1}} X_{1}(t) \leq h_{1}, X_{1}\left(t_{1}\right) \leq k_{1}, X_{2}\left(t_{1}\right) \leq k_{2}, \sup _{t_{1} \leq t \leq t_{2}} X_{2}(t) \leq h_{2}, X_{2}\left(t_{2}\right) \leq k_{3}\right),
$$

where

$$
X_{1}(t)=\ln \left(\frac{S_{1}(t)}{S_{1}\left(t_{0}\right)}\right), \quad X_{2}(t)=\ln \left(\frac{S_{2}(t)}{S_{2}\left(t_{0}\right)}\right)
$$

For a fixed $t \geq 0, X_{1}(t)$ and $X_{2}(t)$ are absolutely continuous random variables, hence one can express (A.1) as the following triple integral:

$$
\int_{x_{1}=-\infty}^{k_{1}} \int_{x_{2}=-\infty}^{k_{2}} \int_{x_{3}=-\infty}^{k_{3}} \mathbb{P}\left(\begin{array}{l}
\sup _{0 \leq t \leq t_{1}} X_{1}(t) \leq h_{1}, X_{1}\left(t_{1}\right) \in d x_{1}, X_{2}\left(t_{1}\right) \in d x_{2}, \\
\sup _{t_{1} \leq t \leq t_{2}} X_{2}(t) \leq h_{2}, X_{2}\left(t_{2}\right) \in d x_{3}
\end{array}\right) d x_{3} d x_{2} d x_{1} .
$$


By conditioning and using the Markov property of processes $X_{1}(t)$ and $X_{2}(t),($ A.3) becomes

$$
\int_{x_{1}=-\infty}^{k_{1}} \int_{x_{2}=-\infty}^{k_{2}} \int_{x_{3}=-\infty}^{k_{3}} P_{1}\left(x_{1}\right) P_{2}\left(x_{1}, x_{2}\right) P_{3}\left(x_{2}, x_{3}\right) d x_{3} d x_{2} d x_{1}
$$

where

$$
\begin{gathered}
P_{1}\left(x_{1}\right)=\mathbb{P}\left(\sup _{0 \leq t \leq t_{1}} X_{1}(t) \leq h_{1}, X_{1}\left(t_{1}\right) \in d x_{1}\right), \\
P_{2}\left(x_{1}, x_{2}\right)=\mathbb{P}\left(X_{2}\left(t_{1}\right) \in d x_{2} \mid X_{1}\left(t_{1}\right) \in d x_{1}\right), \\
P_{3}\left(x_{2}, x_{3}\right)=\mathbb{P}\left(\sup _{t_{1} \leq t \leq t_{2}} X_{2}(t) \leq h_{2}, X_{2}\left(t_{2}\right) \in d x_{3} \mid X_{2}\left(t_{1}\right) \in d x_{2}\right) .
\end{gathered}
$$

The functions $P_{1}\left(x_{1}\right)$ and $P_{2}\left(x_{1}, x_{2}\right)$ are classical results. To obtain the function $P_{3}\left(x_{2}, x_{3}\right)$, the following formula is used (Guillaume [6]):

$$
\begin{aligned}
& \mathbb{P}\left(X_{2}\left(t_{1}\right) \leq x_{2}, \sup _{t_{1} \leq t \leq t_{2}} X_{2}(t) \leq h_{2}, X_{2}\left(t_{2}\right) \leq x_{3}\right) \\
& \quad=N_{2}\left[\frac{x_{2}-\mu_{2} t_{1}}{\sigma_{2} \sqrt{t_{1}}}, \frac{x_{3}-\mu_{2} t_{2}}{\sigma_{2} \sqrt{t_{2}}} ; \sqrt{\frac{t_{1}}{t_{2}}}\right]-\exp \left(\frac{2 \mu_{2}}{\sigma_{2}^{2}} h_{2}\right) N_{2}\left[\frac{x_{2}+\mu_{2} t_{1}}{\sigma_{2} \sqrt{t_{1}}}, \frac{x_{3}-2 h_{2}-\mu_{2} t_{2}}{\sigma_{2} \sqrt{t_{2}}} ;-\sqrt{\frac{t_{1}}{t_{2}}}\right],
\end{aligned}
$$

where $N_{2}[\cdot, \cdot ; \rho]$ is the bivariate standard normal cumulative distribution function with correlation coefficient $\rho$. Differentiating (A.6) twice with respect to variables $x_{2}$ and $x_{3}$, and applying the definition of conditional probability yields

$$
P_{3}\left(x_{2}, x_{3}\right)=\left(\begin{array}{l}
\frac{\exp \left(-(1 / 2)\left(\left(x_{3}-x_{2}-\mu_{2}\left(t_{2}-t_{1}\right)\right) / \sigma_{2} \sqrt{t_{2}-t_{1}}\right)^{2}\right)}{\sigma_{2} \sqrt{2 \pi\left(t_{2}-t_{1}\right)}} \\
-\frac{\exp \left(\left(2 \mu_{2} / \sigma_{2}^{2}\right)\left(h_{2}-x_{2}\right)-(1 / 2)\left(\left(x_{3}+x_{2}-2 h_{2}-\mu_{2}\left(t_{2}-t_{1}\right)\right) / \sigma_{2} \sqrt{t_{2}-t_{1}}\right)^{2}\right)}{\sigma_{2} \sqrt{2 \pi\left(t_{2}-t_{1}\right)}}
\end{array}\right) .
$$

Then, the most cumbersome part of the proof consists in performing the necessary calculations to solve (A.4) in closed form and find the sum of trivariate standard normal cumulative distribution functions given by Proposition 2.1.

\section{B. Proof of Proposition 2.2}

Proof is outlined for $P_{\text {[up-and-up] }}\left(H_{1}, H_{2}, K_{1}, K_{2}, K_{3}, K_{4}, t_{1}, t_{2}, t_{3}\right)$ only. The other distributions are obtained in a similar manner. 
The following lemma is instrumental in proving Proposition 2.2.

Lemma B.1. Let $X_{1}, X_{2}$, and $X_{3}$ be three standard normal random variables defined on a probability space with measure $\mathbb{P}$. Denote by $\theta_{a \cdot b}$ the correlation coefficient between $X_{a}$ and $X_{b},(a, b) \in\{1,2,3\}$. Then, the joint density function of $\left(X_{1}, X_{2}, X_{3}\right)$ is given by

$$
\begin{aligned}
\mathbb{P}\left(X_{1} \in\right. & \left.d x_{1}, X_{2} \in d x_{2}, X_{3} \in d x_{3}\right) \\
= & \exp \left(-\frac{x_{1}^{2}}{2}-\frac{1}{2 \phi_{2 \mid 1}^{2}}\left(x_{2}-\theta_{1 \cdot 2} x_{1}\right)^{2}-\frac{1}{2 \phi_{3 \mid 1 \cdot 2}^{2}}\left(x_{3}-\theta_{1 \cdot 3} x_{1}-\theta_{2 \cdot 3 \mid 1}\left(\frac{x_{2}-\theta_{1 \cdot 2} x_{1}}{\phi_{2 \mid 1}}\right)\right)^{2}\right) \\
& \times\left((2 \pi)^{3 / 2} \phi_{2 \mid 1} \phi_{3 \mid 1 \cdot 2}\right)^{-1},
\end{aligned}
$$

where $\phi_{2 \mid 1}=\sqrt{1-\theta_{1 \cdot 2}^{2}}$ is the standard deviation of $X_{2}$ conditional on $X_{1}, \theta_{2 \cdot 3 \mid 1}=\left(\theta_{2 \cdot 3}-\theta_{1 \cdot 2} \theta_{1 \cdot 3}\right) / \phi_{2 \mid 1}$ is the partial correlation between $X_{2}$ and $X_{3}$ conditional on $X_{1}$, and $\phi_{3 \mid 1 \cdot 2}=\sqrt{1-\theta_{1 \cdot 3}^{2}-\theta_{2 \cdot 3 \mid 1}^{2}}$ is the standard deviation of $X_{3}$ conditional on $X_{1}$ and on $X_{2}$.

Proof of Lemma B.1 is simple. It is known that $X_{2}$ is equal in law to $\theta_{1 \cdot 2} X_{1}+\phi_{2 \mid 1} Y_{2}$, where $Y_{2}$ is a standard normal random variable uncorrelated with $X_{1}$. By applying Cholesky's decomposition to the correlation matrix associated with $\left(X_{1}, X_{2}, X_{3}\right)$, one also finds that $X_{3}$ admits the following representation:

$$
X_{3}=\theta_{1 \cdot 3} X_{1}+\theta_{2 \cdot 3 \mid 1} Y_{2}+\phi_{3 \mid 1 \cdot 2} Y_{3} \longleftrightarrow X_{3}=\theta_{1 \cdot 3} X_{1}+\theta_{2 \cdot 3 \mid 1} \frac{X_{2}-\theta_{1 \cdot 2} X_{1}}{\phi_{2 \mid 1}}+\phi_{3 \mid 1 \cdot 2} Y_{3},
$$

where $Y_{3}$ is a standard normal random variable uncorrelated with $X_{1}$ and $Y_{2}$.

Thus, given $X_{1}, X_{2}$, and $X_{3}$ is normally distributed with mean $\theta_{1 \cdot 3} X_{1}+\theta_{2 \cdot 3 \mid 1}\left(\left(X_{2}-\right.\right.$ $\left.\left.\theta_{1.2} X_{1}\right) / \phi_{2 \mid 1}\right)$ and standard deviation $\phi_{3 \mid 1.2}$. Hence, the density function of $X_{3}$ conditional on $X_{1}$ and $X_{2}$ writes

$$
\begin{aligned}
\mathbb{P}\left(X_{3}\right. & \left.\in d x_{3} \mid X_{1} \in d x_{1}, X_{2} \in d x_{2}\right) \\
& =\exp \left(-\frac{1}{2 \phi_{3 \mid 1 \cdot 2}^{2}}\left(x_{3}-\theta_{1 \cdot 3} x_{1}-\theta_{2 \cdot 3 \mid 1}\left(\frac{x_{2}-\theta_{1 \cdot 2} x_{1}}{\phi_{2 \mid 1}}\right)\right)^{2}\right)\left(\phi_{3 \mid 1 \cdot 2} \sqrt{2 \pi}\right)^{-1},
\end{aligned}
$$

which suffices to prove Lemma B.1.

Next, we proceed to outline proof of Proposition 2.2. By taking the logarithms of the asset prices at the times $t_{1}, t_{2}, t_{3}$, and integrating with respect to these variables, the sought cumulative distribution function becomes

$$
\int_{x_{1}=-\infty}^{k_{1}} \int_{x_{2}=-\infty}^{k_{2}} \int_{x_{3}=-\infty}^{k_{3}} \int_{x_{4}=-\infty}^{k_{4}} \mathbb{P}\left(\begin{array}{c}
\sup _{0 \leq t \leq t_{1}} X_{1}(t) \leq h_{1}, X_{1}\left(t_{1}\right) \in d x_{1}, X_{2}\left(t_{1}\right) \in d x_{2}, \\
\sup _{t_{1} \leq t \leq t_{2}} X_{2}(t) \leq h_{2}, X_{2}\left(t_{2}\right) \in d x_{3}, X_{3}\left(t_{3}\right) \in d x_{4}
\end{array}\right) d x_{4} d x_{3} d x_{2} d x_{1}
$$


where $X_{3}(t)=\ln \left(S_{3}(t) / S_{3}\left(t_{0}\right)\right)$ and the other terms are defined in the proof of Proposition 2.1. Then, by conditioning and using the Markov property of the processes $X_{1}(t), X_{2}(t)$ and $X_{3}(t)$, (B.4) becomes

$$
\int_{x_{1}=-\infty}^{k_{1}} \int_{x_{2}=-\infty}^{k_{2}} \int_{x_{3}=-\infty}^{k_{3}} \int_{x_{4}=-\infty}^{k_{4}} P_{1}\left(x_{1}\right) P_{2}\left(x_{1}, x_{2}\right) P_{3}\left(x_{2}, x_{3}\right) P_{4}\left(x_{1}, x_{3}, x_{4}\right) d x_{4} d x_{3} d x_{2} d x_{1},
$$

where

$$
\begin{gathered}
P_{1}\left(x_{1}\right)=\mathbb{P}\left(\sup _{0 \leq t \leq t_{1}} X_{1}(t) \leq h_{1}, X_{1}\left(t_{1}\right) \in d x_{1}\right), \\
P_{2}\left(x_{1}, x_{2}\right)=\mathbb{P}\left(X_{2}\left(t_{1}\right) \in d x_{2} \mid X_{1}\left(t_{1}\right) \in d x_{1}\right), \\
P_{3}\left(x_{2}, x_{3}\right)=\mathbb{P}\left(\sup _{t_{1} \leq t \leq t_{2}} X_{2}(t) \leq h_{2}, X_{2}\left(t_{2}\right) \in d x_{3} \mid X_{2}\left(t_{1}\right) \in d x_{2}\right), \\
P_{4}\left(x_{1}, x_{3}, x_{4}\right)=\mathbb{P}\left(X_{3}\left(t_{3}\right) \in d x_{4} \mid X_{2}\left(t_{2}\right) \in d x_{3}, X_{1}\left(t_{1}\right) \in d x_{1}\right) .
\end{gathered}
$$

The functions $P_{1}\left(x_{1}\right), P_{2}\left(x_{1}, x_{2}\right)$, and $P_{3}\left(x_{2}, x_{3}\right)$ are dealt with in the proof of Proposition 2.1. The function $P_{4}\left(x_{1}, x_{3}, x_{4}\right)$ is now derived. Since the processes $\left\{S_{1}(t), t \geq 0\right\},\left\{S_{2}(t), t \geq 0\right\}$ and $\left\{S_{3}(t), t \geq 0\right\}$ are geometric Brownian motions with constant drift and diffusion coefficients, the random variables $X_{1}\left(t_{1}\right), X_{2}\left(t_{2}\right)$, and $X_{3}\left(t_{3}\right)$ are normally distributed. Elementary calculations yield their cross-correlations. Applying Cholesky's decomposition to $X_{2}\left(t_{2}\right)$ and $X_{3}\left(t_{3}\right)$, one can see that any linear combination $a_{1} X_{1}\left(t_{1}\right)+a_{2} X_{2}\left(t_{2}\right)+a_{3} X_{3}\left(t_{3}\right)$ for any set of real numbers $\left\{a_{1}, a_{2}, a_{3}\right\}$ is a sum of three uncorrelated normal random variables; hence, it is normally distributed. Therefore, using Fréchet's characterization of multivariate normality (Fréchet [19]), the joint density function $P_{4}\left(x_{1}, x_{3}, x_{4}\right)$ is trivariate normal, and Lemma B.1 yields

$$
\begin{aligned}
P_{4}\left(x_{1}, x_{3}, x_{4}\right)= & \exp \left(-\frac{1}{2 \phi_{4 \mid 1 \cdot 3}^{2}}\left(\varphi_{3}\left(x_{4}\right)-\theta_{1 \cdot 4} \varphi_{1}\left(x_{1}\right)-\theta_{3 \cdot 4 \mid 1}\left(\frac{\varphi_{2}\left(x_{3}\right)-\theta_{1 \cdot 3} \varphi_{1}\left(x_{1}\right)}{\phi_{3 \mid 1}}\right)\right)^{2}\right) \\
& \times\left(\phi_{4 \mid 1 \cdot 3} \phi_{3 \mid 1} \sigma_{1} \sigma_{2} \sigma_{3} \sqrt{t_{1} t_{2} t_{3} 8 \pi^{3}}\right)^{-1},
\end{aligned}
$$

where the notations are defined as follows:

$$
\begin{gathered}
\varphi_{1}\left(x_{1}\right)=\frac{x_{1}-\mu_{1} t_{1}}{\sigma_{1} \sqrt{t_{1}}}, \quad \varphi_{2}\left(x_{3}\right)=\frac{x_{3}-\mu_{2} t_{2}}{\sigma_{2} \sqrt{t_{2}}}, \quad \varphi_{3}\left(x_{4}\right)=\frac{x_{4}-\mu_{3} t_{3}}{\sigma_{3} \sqrt{t_{3}}}, \\
\theta_{1 \cdot 3}=\sqrt{\frac{t_{1}}{t_{2}}} \rho_{1 \cdot 2}, \quad \theta_{1 \cdot 4}=\sqrt{\frac{t_{1}}{t_{3}}} \rho_{1 \cdot 3}, \quad \theta_{3 \cdot 4}=\sqrt{\frac{t_{2}}{t_{3}}} \rho_{2 \cdot 3}, \\
\phi_{3 \mid 1}=\sqrt{1-\theta_{1 \cdot 3}^{2}}, \quad \theta_{3 \cdot 4 \mid 1}=\frac{\theta_{3 \cdot 4}-\theta_{1 \cdot 3} \theta_{1 \cdot 4}}{\phi_{3 \mid 1}}, \quad \phi_{4 \mid 1 \cdot 3}=\sqrt{1-\theta_{1 \cdot 4}^{2}-\theta_{3 \cdot 4 \mid 1}^{2}} .
\end{gathered}
$$


Then, the most cumbersome part of the proof consists in performing the necessary calculations to solve (B.5) in closed form and find the sum of functions given by Proposition 2.2.

\section{Sketch of Proof of Propositions 3.1 and 3.2}

Once endowed with Proposition 2.1, proof of Proposition 3.1 is straightforward, as an application of option pricing theory (Harrison and Pliska [20]) and Girsanov's theorem in two dimensions. The substitutions $\alpha_{1}=v_{1}$ and $\alpha_{2}=v_{2}$ in Proposition 2.1 derive from the introduction of a new measure, which is equivalent to the risk-neutral measure, under which the market numeraire is asset $S_{2}$. If we denote by $\left\{B_{1}(t), t \geq 0\right\}$ and $\left\{B_{2}(t), t \geq 0\right\}$ the two correlated Brownian motions driving $\left\{S_{1}(t), t \geq 0\right\}$ and $\left\{S_{2}(t), t \geq 0\right\}$ under the risk-neutral measure, then the processes $\left\{B_{1}(t)-\sigma_{2} \rho_{1 \cdot 2} t, t \geq 0\right\}$ and $\left\{B_{2}(t)-\sigma_{2} \sqrt{1-\rho_{1 \cdot 2}^{2}} t, t \geq 0\right\}$ are independent standard Brownian motions under the new measure.

Similarly, once endowed with Proposition 2.2, proof of Proposition 3.2 is straightforward, as an application of option pricing theory and Girsanov's theorem in three dimensions. The substitutions $\alpha_{1}=v_{1}, \alpha_{2}=v_{2}$, and $\alpha_{3}=v_{3}$ in Proposition 2.2 derive from the introduction of a new measure, which is equivalent to the risk-neutral measure, under which the market numeraire is asset $S_{3}$. If we denote by $\left\{B_{1}(t), t \geq 0\right\},\left\{B_{2}(t), t \geq 0\right\}$, and $\left\{B_{3}(t), t \geq 0\right\}$ the three correlated Brownian motions driving $\left\{S_{1}(t), t \geq 0\right\},\left\{S_{2}(t), t \geq 0\right\}$, and $\left\{S_{3}(t), t \geq 0\right\}$ under the risk-neutral measure, then the three following processes:

$$
\begin{gathered}
\left\{B_{1}(t)-\sigma_{3} \rho_{1 \cdot 3} t, t \geq 0\right\}, \\
\left\{B_{2}(t)-\sigma_{3}\left(\frac{\rho_{2 \cdot 3}-\rho_{1 \cdot 2} \rho_{1 \cdot 3}}{\sqrt{1-\rho_{1 \cdot 2}^{2}}}\right) t, t \geq 0\right\}, \\
\left\{B_{3}(t)-\sigma_{3} t \sqrt{1-\rho_{1 \cdot 3}^{2}-\left(\frac{\left(\rho_{2 \cdot 3}-\rho_{1 \cdot 2} \rho_{1 \cdot 3}\right)^{2}}{1-\rho_{1 \cdot 2}^{2}}\right)}, t \geq 0\right\}
\end{gathered}
$$

are independent standard Brownian motions under the new measure.

\section{References}

[1] D. Freedman, Brownian Motion and Diffusion, Holden-Day, San Francisco, Calif, USA, 1971.

[2] L. Barba Escribá, "A stopped Brownian motion formula with two sloping line boundaries," The Annals of Probability, vol. 15, no. 4, pp. 1524-1526, 1987.

[3] P. Salminen, "On the first hitting time and the last exit time for a Brownian motion to/from a moving boundary," Advances in Applied Probability, vol. 20, no. 2, pp. 411-426, 1988.

[4] N. Kunitomo and M. Ikeda, "Pricing options with curved boundaries," Mathematical Finance, vol. 2, no. 4, pp. 275-298, 1992.

[5] A. N. Borodin and P. Salminen, Handbook of Brownian Motion-Facts and Formulae, Probability and Its Applications, Birkhäuser, Basel, Switzerland, 2nd edition, 2002.

[6] T. Guillaume, "Analytical valuation of options on joint minima and maxima," Applied Mathematical Finance, vol. 8, no. 4, pp. 209-233, 2001. 
[7] S. Iyengar, "Hitting lines with two-dimensional Brownian motion," SIAM Journal on Applied Mathematics, vol. 45, no. 6, pp. 983-989, 1985.

[8] H. He, W. P. Keirstead, and J. Rebholz, "Double lookbacks," Mathematical Finance, vol. 8, no. 3, pp. 201-228, 1998.

[9] T. Guillaume, “Step double barrier options," Journal of Derivatives, vol. 18, no. 1, pp. 59-79, 2010.

[10] A. Genz, "Numerical computation of rectangular bivariate and trivariate normal and t probabilities," Statistics and Computing, vol. 14, no. 3, pp. 251-260, 2004.

[11] R. C. Heynen and H. M. Kat, "Partial barrier options," Journal of Financial Engineering, vol. 3, pp. 253-274, 1994.

[12] G. F. Armstrong, "Valuation formulae for window barrier options," Applied Mathematical Finance, vol. 8, no. 4, pp. 197-209, 2001.

[13] T. Guillaume, "Window double barrier options," Review of Derivatives Research, vol. 6, no. 1, pp. 47-75, 2003.

[14] R. C. Heynen and H. M. Kat, “Crossing barriers,” Risk Magazine, vol. 7, 1994.

[15] Y. Kwok, L. Wu, and H. Yu, "Pricing multi-asset options with an external barrier," International Journal of Theoretical and Applied Finance, vol. 1, no. 4, pp. 523-541, 1998.

[16] H. Y. Wong and Y.-K. Kwok, "Multi-asset barrier options and occupation time derivatives," Applied Mathematical Finance, vol. 10, no. 3, pp. 245-266, 2003.

[17] E. G. Haug, The Complete Guide to Option Pricing Formulas, McGraw Hill, 2007.

[18] P. Glasserman, Monte Carlo Methods in Financial Engineering, vol. 53 of Applications of Mathematics, Springer, New York, NY, USA, 2004.

[19] M. Fréchet, "Généralisations de la loi de probabilité de Laplace," Annales de l'Institut Henri Poincaré, vol. 12, pp. 1-29, 1951.

[20] J. M. Harrison and S. R. Pliska, "Martingales and stochastic integrals in the theory of continuous trading," Stochastic Processes and their Applications, vol. 11, no. 3, pp. 215-260, 1981. 


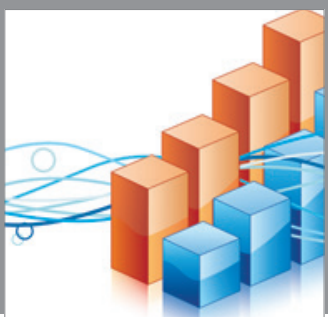

Advances in

Operations Research

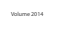

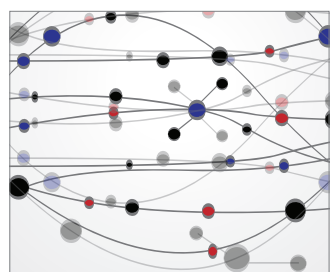

\section{The Scientific} World Journal
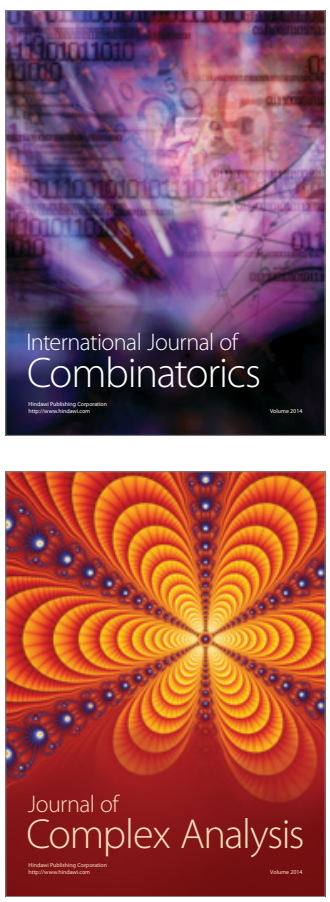

International Journal of

Mathematics and

Mathematical

Sciences
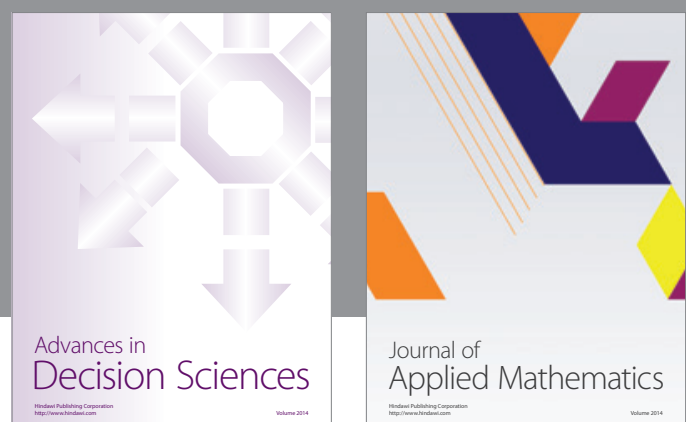

Journal of

Applied Mathematics
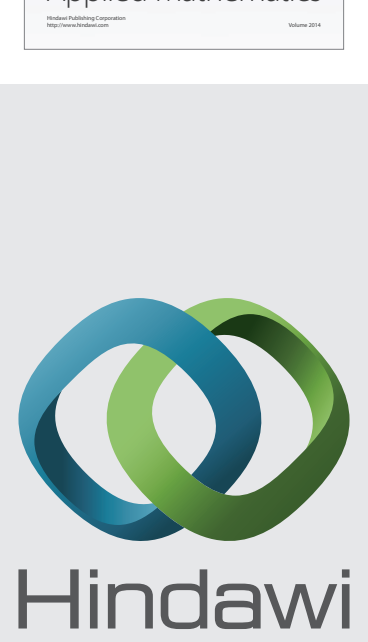

Submit your manuscripts at http://www.hindawi.com
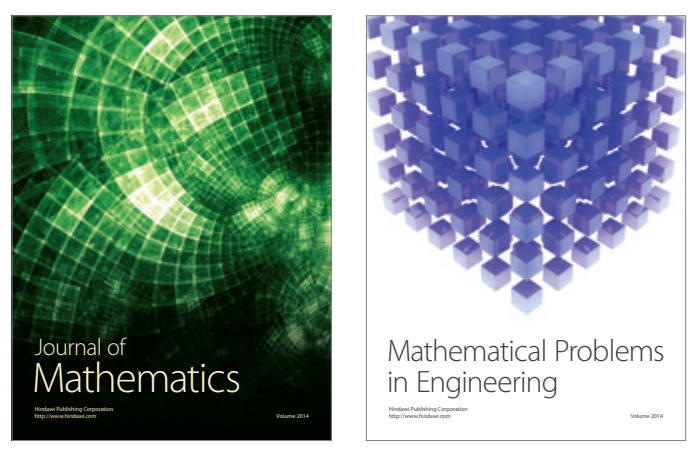

Mathematical Problems in Engineering
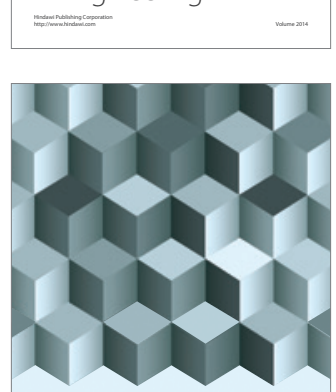

Journal of

Function Spaces
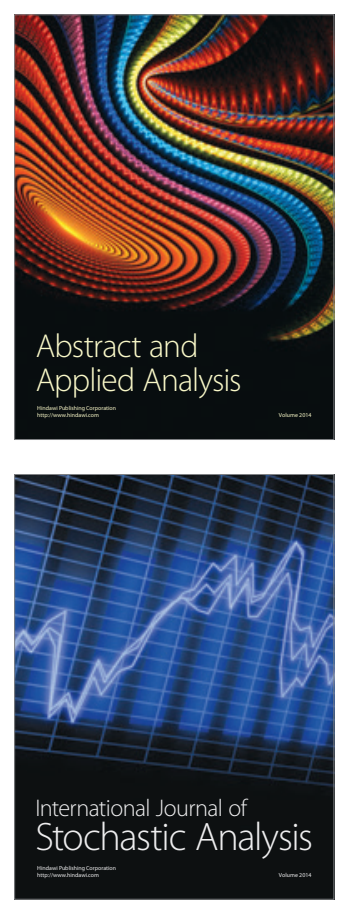

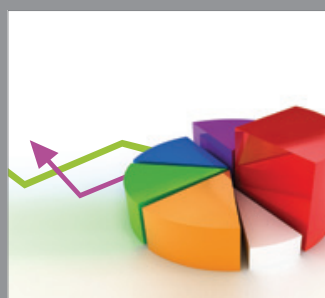

ournal of

Probability and Statistics

Promensencen
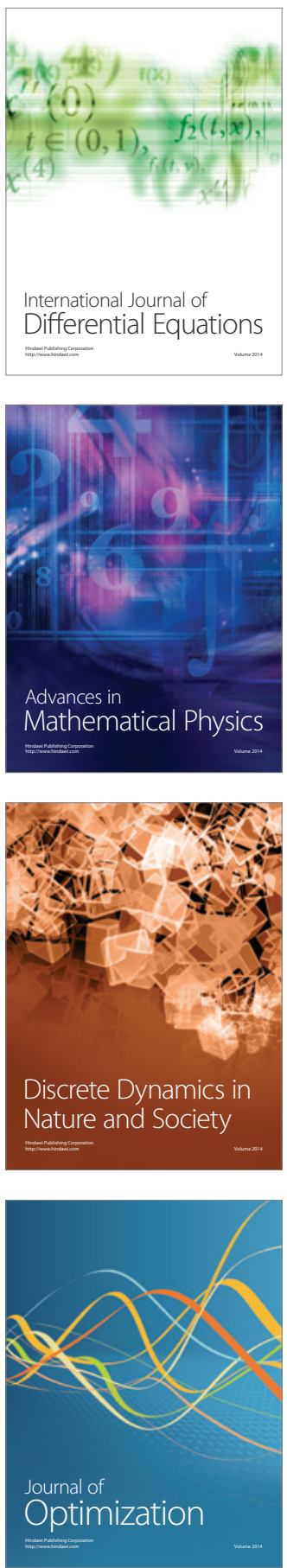